\title{
Excitonic coherence in a confined lattice : a simple model to highlight the relevance of the perturbation theory
}

\author{
Vincent Pouthier* \\ Institut UTINAM, Université de Franche-Comté, \\ CNRS UMR 6213, 25030 Besançon Cedex, France
}

(Dated: February 25, 2011)

\begin{abstract}
The exciton-phonon system in a confined environment is revisited within standard perturbation theory. Special attention is paid for describing the time evolution of the excitonic coherences at finite temperature. To proceed, the system involving an exciton dressed by a single phonon mode is considered. Due to its simplicity, it is solved exactly so that the relevance of the perturbation theory is checked. Within the nonadiabatic weak coupling limit, it is shown that several time scales govern the coherence dynamics. In the short time limit, the coherences behave as if the exciton was insensitive to the phonon bath. Then, quantum decoherence takes place. Finally the coherences recur almost periodically at specific revival times. Coherence decay and revival strongly depend on the nature of the excitonic states. In particular, for odd lattice sizes, the coherence of the state exactly located at the band center survives over a very long time scale. Therefore, confinementinduced decoherence softening favors high fidelity quantum state transfer and allows to encode the information on a quantum bit almost insensitive to quantum decoherence.
\end{abstract}

PACS numbers: 03.65.Yz,63.22.-m,71.35.-y,71.38.-k

\section{INTRODUCTION}

Molecular lattices, large molecules and biopolymers exhibit regularly distributed atomic subunits. Due to dipole-dipole interaction, the energy of a specific electronic transition, or a high frequency vibrational mode, delocalizes between these subunits. It gives rise to narrow-band excitons able to move coherently and that play a key role in understanding various phenomena ${ }^{1,2}$. In light-harvesting complexes, Frenkel excitons propagating along the array of pigment proteins allow the conversion of solar energy into chemical energy ${ }^{3-7}$. In $\alpha$-helices, amide-I excitons promoting vibrational energy flow may participate in the transduction of the chemical energy into mechanical work ${ }^{8-19}$. In adsorbed molecular nanostructures, vibrons provide an alternative to nanoscale electronics and enhance surface reactions ${ }^{20-29}$. Finally, since quantum bits may be encoded in high frequency vibrational modes ${ }^{30-32}$, vibron-mediated quantum state transfer is a promising way for quantum information processing $^{33}$. Unfortunately, the exciton does not propagate freely but it interacts with the phonons of the host medium $^{34,35}$. The phonons are responsible for dephasing and they tend to destroy the coherent nature of the exciton.

So defined, the exciton-phonon system is a prototype of an open system (exciton) coupled with a thermal bath (phonons) $)^{2,36,37}$. Therefore, information about the exciton properties is encoded in the reduced density matrix (RDM) whose dynamics is governed by a generalized master equation (GME). Among the different strategies elaborated to derive exact GME, the time-convolutionless method (TCL-GME) plays a central role ${ }^{37-42}$. Within this method, a specific projector is used to obtain a time local GME in which the influence of the bath is encoded in a time-dependent relaxation operator. Its form is par- ticularly suitable for describing non-Markovian processes and for building approximate equations through a systematic perturbation expansion.

In many situations, narrow-band excitons propagate slower than the phonons and the nonadiabatic weak coupling limit is reached. The Born approximation is invoked and a second order expansion is performed to derive a $\mathrm{TCL}_{2}$-GME. The relaxation operator is thus defined in terms of the exciton-phonon coupling correlation functions whose dynamics is governed by free phonons. In an infinite lattice, the phonons behave as a reservoir insensitive to the exciton ${ }^{43}$. The correlation functions vanish after a short time scale and the Markov limit is reached. The $\mathrm{TCL}_{2}$-GME becomes a system of first order differential equations with constant coefficients whose solution provides information about the exciton properties (coherence dynamics ${ }^{18}$, coherence transfer ${ }^{44}$, diffusion coefficient $\left.{ }^{16,17,45} \ldots\right)$.

In a finite size lattice, a different behavior takes place due to the discrete nature of the phonon energy spectrum $^{46,47}$. As shown recently ${ }^{48}$, the confinement yields the occurrence of quantum recurrences. These recurrences provide to the relaxation operator an almost periodic nature whose main contribution results from the coupling with the lowest frequency phonon mode (LFPM). The GME reduces to a linear system of differential equations with almost periodic coefficients. In accordance with the Floquet theory, unstable solutions emerge for particular values of the model parameters. They originate in parametric resonances between specific excitonic frequencies and the frequencies of the relaxation operator that involve the LFPM. The parametric resonances give rise to an unphysical exponential growth of the RDM indicating that $\mathrm{TCL}_{2}$-GME breaks down. Moreover, outside parametric resonances, the $\mathrm{TCL}_{2}$-GME fails in reproducing the dynamics. 
To overcome the problems inherent in the nonMarkovian dynamics, different strategies have be elaborated. Examples among many are the correlated projection superoperators method ${ }^{49-51}$, the time-dependent projection-operator approach ${ }^{52-54}$, the effective-mode representation of non-Markovian dynamics ${ }^{55,56}$, the nonperturbative quantum master equation ${ }^{57,58}$, the continuous fraction method m9-61 $^{59}$ and the quantum jumps approach $^{62-64}$. In the present paper, the non-Markovian exciton-phonon dynamics is revisited within the standard perturbation theory $(\mathrm{PT})^{65,66}$. Following recent works, it will be applied for describing a confined exciton coupled with acoustic phonons, i.e. the so-called Fröhlich model ${ }^{46-48}$. Special attention will be paid to characterize the excitonic coherences. They measure the ability of the exciton to develop superimpositions involving the vacuum and the one-exciton states and they play a key role in understanding various phenomena (optical response ${ }^{67}$, quantum state transfer $\left.{ }^{68} \ldots\right)$. The aim of this work is to show that $\mathrm{PT}$ is more accurate than $\mathrm{TCL}_{2}$-GME provided that the lattice size is not too large. To proceed, we shall restrict our attention to the system formed by the exciton dressed by the LFPM, only. The generalization to the coupling with all the phonon modes will be addressed in a forthcoming paper. Our motivation is twofold. First, the system "exciton+LFPM" is so simple that numerical simulations can be carried out easily. Consequently, it will be straightforward to check the relevance of PT. Second, treating the system "exciton+ LFPM" may appear as a first step to renormalize the influence of this mode and to avoid instabilities. In a second step, standard open system theories may be apply, the remaining phonon modes being viewed as a new thermal bath.

The paper is organized as follows. In Sec. II, the exciton-phonon Hamiltonian is described and the excitonic coherences are defined. Then, PT is applied to evaluate the eigenenergies and the eigenstates of the system. An approximate expression for the coherences is finally established. In Sec. III, a numerical analysis is performed in which PT is compared with exact calculations. These results are discussed and interpreted in Sec. IV.

\section{THEORETICAL BACKGROUND}

\section{A. Model Hamiltonian}

The Fröhlich model in a finite size lattice with $N$ sites $x=1, \ldots, N$ has been described in numerous papers ${ }^{46-48}$. We only give here a brief summary with a special emphasis on the coupling with the LFPM.

Within this model, one-exciton states are the first excited states of $N$ coupled two-level systems. They define $N$ superimpositions of incident and reflected plane waves with quantized wave vectors $k \pi / L$, with $k=1, . ., N$ and
$L=N+1$. They are expressed as

$$
|k\rangle=\sum_{x=1}^{N} \sqrt{\frac{2}{L}} \sin \left(\frac{k \pi x}{L}\right)|x\rangle,
$$

where $|x\rangle$ is the first excited state of the $x$ th twolevel system. The corresponding eigenenergies $\omega_{k}=$ $\omega_{0}+2 \Phi \cos (k \pi / L)$ involve the Bohr frequency of each two-level system $\omega_{0}$ and the hopping constant between neighboring sites $\Phi(\hbar=1$ will be used throughout this paper). The exciton Hamiltonian is thus written as : $H_{A}=\sum_{k=1}^{N} \omega_{k}|k\rangle\langle k|$. Note that the vacuum state $|\oslash\rangle$ describes all the two-level systems in their ground state with zero energy. The phonons refer to the external motions of the lattice sites that behave as point masses $M$ connected via force constants $W$. They define $N$ normal modes with wave vectors $p \pi / L$ and frequencies $\Omega_{p}=\Omega_{c} \sin (p \pi / 2 L)$, with $p=1, . ., N$ and $\Omega_{c}=\sqrt{4 W / M}$. The dynamics of the LFPM $p=1$ is governed by the Hamiltonian $H_{B}=\Omega a^{\dagger} a$, where $\Omega \equiv \Omega_{1}, a^{\dagger}$ and $a$ being the standard phonon operators. Finally, the exciton-phonon interaction $V$ results from a stochastic modulation of the energy of each two-level system by the external motions of the lattice. Restricting our attention to the influence of the LFPM, this interaction favors the exciton scattering from $|k\rangle$ to $|k \pm 1\rangle$ mediated by phonon exchanges ${ }^{48}$

As a result, the exciton-phonon Hamiltonian is defined as $H=H_{0}+V$ where $H_{0}=H_{A}+H_{B}$ is the unperturbed Hamiltonian. The coupling $V$ is written as ${ }^{48}$

$$
V=\sum_{k=1}^{N-1} \eta\left(a^{\dagger}+a\right)[|k\rangle\langle k+1|+| k+1\rangle\langle k|],
$$

where $\eta=\left[\left(E_{B} \Omega / L\right)\left(1-\left(\Omega / \Omega_{c}\right)^{2}\right)\right]^{1 / 2}, E_{B}$ being the small polaron binding energy expressed in terms of the coupling strength $\chi$ as $E_{B}=\chi^{2} / W$. Since $H$ conserves the exciton number, the Hilbert space is partitioned into independent subspaces $\mathcal{E}=\mathcal{E}_{0} \oplus \mathcal{E}_{1}$. In the zero-exciton subspace $\mathcal{E}_{0}, V=0$ so that the unperturbed states are eigenstates of $H$. They correspond to tensor products involving the vacuum $|\oslash\rangle$ and the phonon number states $|n\rangle$ and they describe $n$ free phonons with energy $n \Omega$. In the one-exciton subspace $\mathcal{E}_{1}$, the unperturbed states $|k, n\rangle=|k\rangle \otimes|n\rangle$, with energy $\epsilon_{k, n}^{(0)}=\omega_{k}+n \Omega$, refer to $n$ phonons accompanied by an exciton in state $|k\rangle$. Since $V$ turns on in $\mathcal{E}_{1}$, they are no longer eigenstates of $H$. The exact eigenstates $\left|\Psi_{\mu}\right\rangle$, with eigenenergies $\epsilon_{\mu}$, are superimpositions of the unperturbed states.

This model Hamiltonian will be used for describing the exciton dynamics and PT will be applied to evaluate $\left|\Psi_{\mu}\right\rangle$ and $\epsilon_{\mu}$. To proceed, we shall consider the nonadiabatic weak coupling limit: $\omega_{0} \gg \Omega_{c}$ (high energy exciton), $4 \Phi<\Omega_{c}$ (nonadiabatic limit) and $E_{B} \ll \Phi$ (weak coupling limit). As a result, $\Omega>\left|\omega_{k}-\omega_{k \pm 1}\right|, \forall k$ so that there is no resonance between coupled unperturbed states. Nevertheless, both $\eta$ and the Bohr frequencies 
connected to coupled unperturbed states decrease with $L$. Since Bohr frequencies decay faster than $\eta$, quasiresonances occur for large lattice sizes. Consequently, as explained in Sec. II.C., PT can be applied provided that $L$ is not too large.

Although the present model is rather general, it will be used for describing amide-I excitons in $\alpha$-helices, i.e. a situation in which the lattice temperature is about to or higher than the phonon cutoff frequency ${ }^{8-19}$. In $\alpha$ helices, peptide units linked by $\mathrm{H}$ bonds are regularly distributed. Each unit contains a high frequency $\mathrm{C}=\mathrm{O}$ vibration (amide-I mode) that gives rise to vibrational excitons coupled with the phonons of the $\mathrm{H}$ bond network. Such a system is a prototype of a finite size lattice since helices containing from 3 to 15 residues are the most abundant in nature ${ }^{69}$.

Of course, the present model is too simple to accurately describe vibrational energy flow in a real protein whose dynamics exhibits a tremendous complexity due to the rather large number of degrees of freedom. In particular, this model is unable to describe energy relaxation because it conserves the number of amide-I exciton. In proteins, the amide-I lifetime, typically of about 1 ps, results from intramolecular energy redistribution due to the anharmonic coupling between each amide-I mode and a set of intramolecular normal modes whose displacements are strongly localized on the $\mathrm{C}=\mathrm{O}$ groups ${ }^{15,16,70-72}$. Consequently, we do not claim that the model is relevant to explain in details the vibrational dynamics in a protein. Nevertheless, its interest lies in the fact that it provides a simple approach to promote the idea that PT is more accurate than the $\mathrm{TCL}_{2}$-GME method which has been applied recently to a similar model ${ }^{48}$. A more detailed study will be addressed in a forthcoming paper.

\section{B. Excitonic coherences}

Coherences are RDM elements that measure the ability of the exciton to develop superimpositions involving the vacuum and the one-exciton states. As detailed previously ${ }^{18,44,47,48}$, coherences switch on when the exciton-phonon system is brought in a configuration out of equilibrium. To proceed, the exciton is prepared in a superimposition $\left|\psi_{A}\right\rangle=c_{0}|\oslash\rangle+c_{1}\left|k_{1}\right\rangle$, with $\left|c_{0}\right|^{2}+\left|c_{1}\right|^{2}=1$. By contrast, the phonons are assumed to be in thermal equilibrium at temperature $T$. Their properties are encoded in the density matrix $\rho_{B}$ so that the initial state of the whole system is specified by the density matrix $\rho=\left|\psi_{A}\right\rangle\left\langle\psi_{A}\right| \otimes \rho_{B}$.

In the excitonic eigenbasis, the coherence $\sigma_{k_{2} \oslash}(t)$ is thus defined as

$$
\sigma_{k_{2} \oslash}(t)=\left\langle k_{2}\left|\operatorname{Tr}_{B}\left[e^{-i H t} \rho e^{i H t}\right]\right| \oslash\right\rangle,
$$

where $\operatorname{Tr}_{B}$ is a partial trace over the phonon degrees of freedom. After simple algebraic manipulations, the coherence is rewritten as $\sigma_{k_{2} \oslash}(t)=G_{k_{2} k_{1}}(t) \sigma_{k_{1} \oslash}(0)$, with $\sigma_{k_{1} \oslash}(0)=c_{0}^{*} c_{1}$. The matrix element $G_{k_{2} k_{1}}(t)$ is expressed as

$$
G_{k_{2} k_{1}}(t)=\sum_{n=0}^{\infty} P_{n} e^{i n \Omega t}\left\langle k_{2}, n\left|e^{-i H t}\right| k_{1}, n\right\rangle,
$$

where $P_{n}=[1-\exp (-\beta \Omega)] \exp (-\beta n \Omega)$ is the equilibrium population of the $n$th phonon number state $(\beta=$ $1 / k_{B} T, k_{B}$ being the Boltzmann constant). The coherence $\sigma_{k_{2} \oslash}(t)$ measures the ability of the exciton to develop a superimposition involving $|\oslash\rangle$ and $\left|k_{2}\right\rangle$ at time $t$, given that a superimposition involving $|\oslash\rangle$ and $\left|k_{1}\right\rangle$ was initially created. Consequently, $G_{k_{2} k_{1}}(t)$ generalizes the concept of transition amplitude and it defines the effective propagator of the exciton. It yields the probability amplitude to observe the exciton in state $\left|k_{2}\right\rangle$ at time $t$ given that it was in state $\left|k_{1}\right\rangle$ at $t=0$. The effective nature of the propagator results from the fact that the exciton interacts with the phonons during its transition. More precisely, the full system being prepared in the factorized state $\left|k_{1}, n\right\rangle, G_{k_{2} k_{1}}(t)$ measures the probability amplitude to observe the system in a factorized state $\exp (-i n \Omega t)\left|k_{2}, n\right\rangle$ that characterizes an excitonic transition during which the phonons evolve freely. An average over the initial phonon number is finally performed.

In the local basis, the effective propagator gives information about coherence transfer along the lattice. A particular element $G_{x_{2} x_{1}}(t)$ measures the ability of the exciton to develop a superimposition involving the vacuum and the local state $\left|x_{2}\right\rangle$ at time $t$, such a superimposition being initially created on the site $x_{1}$. From Eq.(1), $G_{x_{2} x_{1}}(t)$ is defined as

$$
G_{x_{2} x_{1}}(t)=\sum_{k_{2}=1}^{N} \sum_{k_{1}=1}^{N} u_{k_{2} x_{2}} u_{k_{1} x_{1}} G_{k_{2} k_{1}}(t),
$$

where $u_{k x}=\sqrt{2 / L} \sin (k \pi x / L)$.

The effective propagator is the central object of the present study. It characterizes the way the phonon bath modifies the excitonic coherences in either the eigenbasis or the local basis. Moreover, it provides information about exciton-mediated quantum state transfer. Finally, it yields the spectral response $\left(S(t) \propto \sum_{x_{1}} \sum_{x_{2}} G_{x_{2} x_{1}}(t)\right)$ whose Fourier transform is the exciton absorption spectrum. For the simple system "exciton+LFPM", the numerical diagonalization of $H$ can be carried out. As a result, we are able to build $\left|\Psi_{\mu}\right\rangle$ and $\epsilon_{\mu}$ so that Eqs.(4) and (5) can be computed easily. In Sec. III, these exact calculations will be compared with PT that is presented in the next section.

\section{Perturbation theory}

\section{Eigenenergies and eigenstates}

According to standard $\mathrm{PT}^{65,66}, \epsilon_{\mu}$ and $\left|\Psi_{\mu}\right\rangle$ can be expanded as Taylor series around the unperturbed quan- 
tities as

$$
\begin{aligned}
\epsilon_{k, n} & =\epsilon_{k, n}^{(0)}+\epsilon_{k, n}^{(1)}+\epsilon_{k, n}^{(2)}+\ldots \\
\left|\Psi_{k, n}\right\rangle & =|k, n\rangle+\left|\Psi_{k, n}^{(1)}\right\rangle+\left|\Psi_{k, n}^{(2)}\right\rangle+\ldots
\end{aligned}
$$

where $\epsilon_{k, n}^{(q)}$ and $\left|\Psi_{k, n}^{(q)}\right\rangle$ stand for the $q$ th order corrections. Note that the index $\mu$ now reduces to the indexes $(k, n)$ that refer to unperturbed states.

The eigenenergies up to second order in the excitonphonon coupling are written as $\left(\epsilon_{k, n}^{(1)}=0\right)$

$$
\epsilon_{k, n}=\omega_{k}+\delta \omega_{k}^{(2)}+n\left(\Omega+\delta \Omega_{k}^{(2)}\right)
$$

where $\delta \Omega_{k}^{(2)}$ and $\delta \omega_{k}^{(2)}$ are expressed in terms of $\mu_{k}=$ $1-\delta_{k, N}$ and $\nu_{k}=1-\delta_{k, 1}$ as

$$
\begin{aligned}
\delta \Omega_{k}^{(2)} & =\frac{2 \eta^{2} \mu_{k}\left(\omega_{k}-\omega_{k+1}\right)}{\left(\omega_{k}-\omega_{k+1}\right)^{2}-\Omega^{2}}+\frac{2 \eta^{2} \nu_{k}\left(\omega_{k}-\omega_{k-1}\right)}{\left(\omega_{k}-\omega_{k-1}\right)^{2}-\Omega^{2}} \\
\delta \omega_{k}^{(2)} & =\frac{\eta^{2} \mu_{k}}{\omega_{k}-\omega_{k+1}-\Omega}+\frac{\eta^{2} \nu_{k}}{\omega_{k}-\omega_{k-1}-\Omega} .
\end{aligned}
$$

As shown in Eq. (8), $\delta \omega_{k}^{(2)}$ is the correction of the energy of an exciton in state $|k\rangle$ due to its coupling with the phonons. This correction does not depend on the phonon number. It results from the spontaneous emission of a phonon in the course of which the exciton realizes transitions from $|k\rangle$ to $|k \pm 1\rangle$. However, in the nonadiabatic limit, the energy cannot be conserved during such processes so that the phonon emission is not a real process. The exciton is only able to exchange a virtual phonon which is first emitted and then immediately reabsorbed. In accordance with the small polaron concept, $\delta \omega_{k}^{(2)}$ is the manifestation of the so-called dressing effect. The exciton does not propagate freely but it is dressed by a virtual phonon cloud.

Similarly, $\delta \Omega_{k}^{(2)}$ is the energy correction of a phonon accompanied by an exciton in state $|k\rangle$. As shown in Eq.(7), the energy correction $n \delta \Omega_{k}^{(2)}$ now involves the phonon number and it results from two mechanisms. First, the phonon can be absorbed giving rise to excitonic transitions from $|k\rangle$ to $|k \pm 1\rangle$. Such a process does not conserve the energy within the nonadiabatic limit so that the phonon is immediately re-emitted. Second, the phonon induces the stimulated emission of a second phonon in the course of which the exciton realizes transitions. But, as previously, the emitted phonon is immediately reabsorbed. Both mechanisms correspond to virtual processes indicating that a phonon does no longer evolve freely. It is dressed by an exciton that realizes virtual transitions.

In Appendix A, PT is applied to derive eigenstates up to second order in the exciton-phonon coupling (see Eq.(A1)). Due to this coupling, the eigenstates do no longer factorize and a quantum entanglement occurs. The first order correction to $|k, n\rangle$ involves the neighboring states $|k \pm 1, n \pm 1\rangle$ which are directly coupled through $V$. By contrast, the second order correction exhibits three contributions. The first contribution modifies the weight of the unperturbed state ensuring the normalization of $\left|\Psi_{k, n}\right\rangle$ to unity up to second order. The second contribution involves second nearest neighbor states $|k \pm 2, n \pm 2\rangle$. The last contribution reveals that the eigenstates involve unperturbed states in which either the phonon number or the exciton state is conserved.

At this step, let us discuss the validity of the second order PT. Indeed, PT works quite well provided that the coupling between two unperturbed states $|k, n\rangle$ and $|k \pm 1, n \pm 1\rangle$ is smaller than the corresponding Bohr frequency. To establish a criterion at finite temperature, one first assumes that the phonon number is about the average phonon number $\bar{n}=[\exp (\beta \Omega)-1]^{-1}$. Then, since $\Omega \ll \Omega_{c}$, the coupling strength scales as $\eta \approx \sqrt{E_{B} \Omega / L}$. Finally, the smallest Bohr frequency involving excitonic states close to the band center, it is about $\Omega(1-2 B)$, $B=2 \Phi / \Omega_{c}$ being the adiabaticity. In that context, for $k_{B} T \gg \Omega, \mathrm{PT}$ can be applied provided that

$$
\frac{2}{\pi} \sqrt{\frac{E_{B} k_{B} T}{\Omega_{c}^{2}}} \frac{\sqrt{L}}{1-2 B}<x,
$$

where $x$ specifies the desired accuracy for the approximate eigenenergies (for a two-level system, $x=0.286$ ensures an error lower than 1\%). Eq.(9) defines a critical curve in the parameter space. As a result, for a given temperature and a fixed coupling strength, it reveals that a critical length $L^{*}$ discriminates between two regimes. For $L<L^{*}$, PT is expected to correctly describe the exciton-phonon dynamics whereas it certainly breaks down for $L>L^{*}$. Of course, the smaller the coupling and the temperature are, the longer the critical length is.

\section{Excitonic Coherences}

Inserting Eqs.(7) and (A1) into Eq.(4) yields the effective exciton propagator up to second order in the coupling. It is expressed in terms of the renormalized energies $\hat{\omega}_{k}=\omega_{k}+\delta \omega_{k}^{(2)}$ and $\hat{\Omega}_{k}=\Omega+\delta \Omega_{k}^{(2)}$ as

$$
\begin{aligned}
& G_{k_{2} k_{1}}(t)=\sum_{k=1}^{N} e^{-i \hat{\omega}_{k} t} \sum_{n=0}^{\infty} P_{n} e^{-i n \delta \Omega_{k}^{(2)} t} \sum_{m=0}^{\infty} e^{i(n-m) \hat{\Omega}_{k} t} \\
& {\left[\delta_{k k_{1}} \delta_{k k_{2}} \delta_{n m}+\left\langle k_{2}, n \mid \Psi_{k, m}^{(1)}\right\rangle\left\langle\Psi_{k, m}^{(1)} \mid k_{1}, n\right\rangle\right.} \\
& \left.+\delta_{k k_{1}} \delta_{n m}\left\langle k_{2}, n \mid \Psi_{k, n}^{(2)}\right\rangle+\delta_{k k_{2}} \delta_{n m}\left\langle\Psi_{k, n}^{(2)} \mid k_{1}, n\right\rangle\right] .
\end{aligned}
$$

From the expression of $\left|\Psi_{k, m}^{(1)}\right\rangle$ (Eq.(A1)), $m$ is either equal to $n$ or $n \pm 1$ so that the sum over $m$ simplifies. By contrast, the sum over $n$ defines an average according to a time dependent distribution function written as

$$
P_{n}^{k}(t)=\frac{e^{-n\left(\beta \Omega+i \delta \Omega_{k}^{(2)} t\right)}}{Z_{B}^{k}(t)},
$$




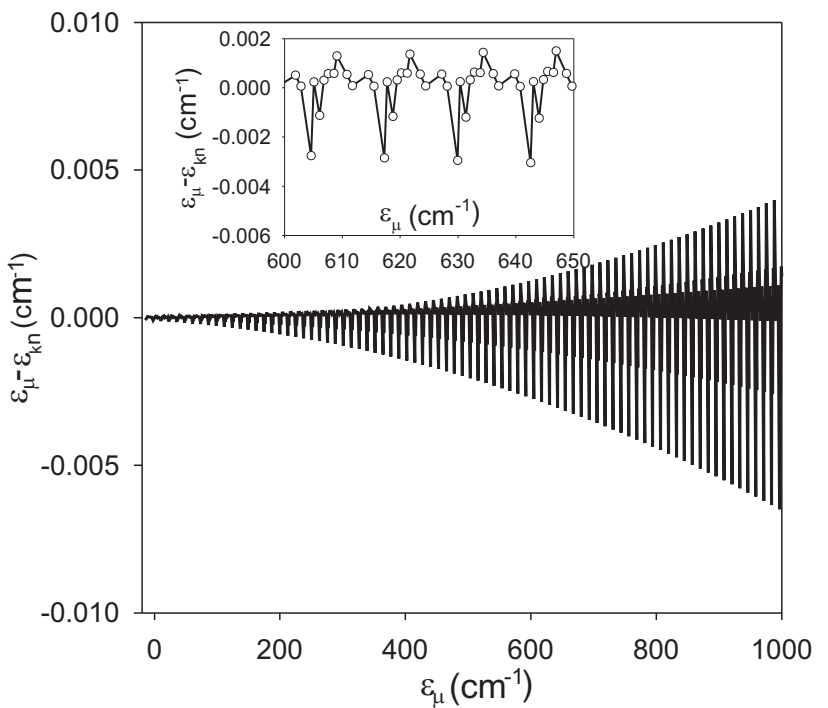

FIG. 1: Energy difference $\epsilon_{\mu}-\epsilon_{k, n}$ versus $\epsilon_{\mu}$ for $N=11$.

where $Z_{B}^{k}(t)=\left[1-\exp \left(-\beta \Omega-i \delta \Omega_{k}^{(2)} t\right)\right]^{-1}$. Strictly speaking, $P_{n}^{k}(t)$ is not a probability distribution since it is a complex-valued function. Nevertheless, it reduces to the equilibrium population of the phonon number states at $t=0$, i.e. $P_{n}^{k}(t=0) \equiv P_{n} \forall k$. In that case, the normalization factor $Z_{B}^{k}(t=0)$ is the equilibrium partition function $Z_{B}=[1-\exp (-\beta \Omega)]^{-1}$.

Since the sum over $k$ simplifies one finally obtains the effective propagator whose expression is displayed in Appendix B.

\section{NUMERICAL RESULTS}

In this section, the previous formalism is applied for describing amide-I excitons in a finite size lattice of $\mathrm{H}$ bonded peptide units ${ }^{18,44,47,48}$. To proceed, typical values for the parameters are used: $\omega_{0}=1660 \mathrm{~cm}^{-1}$, $W=15 \mathrm{Nm}^{-1}, M=1.8 \times 10^{-25} \mathrm{~kg}$ and $\Phi=7.8 \mathrm{~cm}^{-1}$. The phonon cutoff frequency is equal to $\Omega_{c}=96.86$ $\mathrm{cm}^{-1}$ indicating that the nonadiabatic limit is reached $(B=0.16)$. The coupling strength is about $\chi=8$ $\mathrm{pN}\left(E_{B}=0.21 \mathrm{~cm}^{-1}\right)$, i.e. a value close to ab initio estimate $^{73}$. With these parameters, Eq.(9) shows that the critical length $L^{*} \approx 20$ ensures the PT validity over the temperature range $T \in[0,300] \mathrm{K}$.

As shown in Fig. 1, PT yields a very good estimate of the energy spectrum over a large energy scale (up to $\left.1000 \mathrm{~cm}^{-1}\right)$. The smaller the energy is, the better the agreement is. The energy difference $\Delta \epsilon=\epsilon_{\mu}-\epsilon_{k, n}$ increases with $\epsilon_{\mu}$ indicating that the PT accuracy decreases as the phonon number $n$ increases. This feature results from the $n$ dependence of the exciton-phonon coupling that scales as $\sqrt{n}$. For instance, for the eigenstate $\mu=100\left(\epsilon_{100}=108.88 \mathrm{~cm}^{-1}\right)$ that refers to the unperturbed state $(k=4, n=8)$, the energy difference

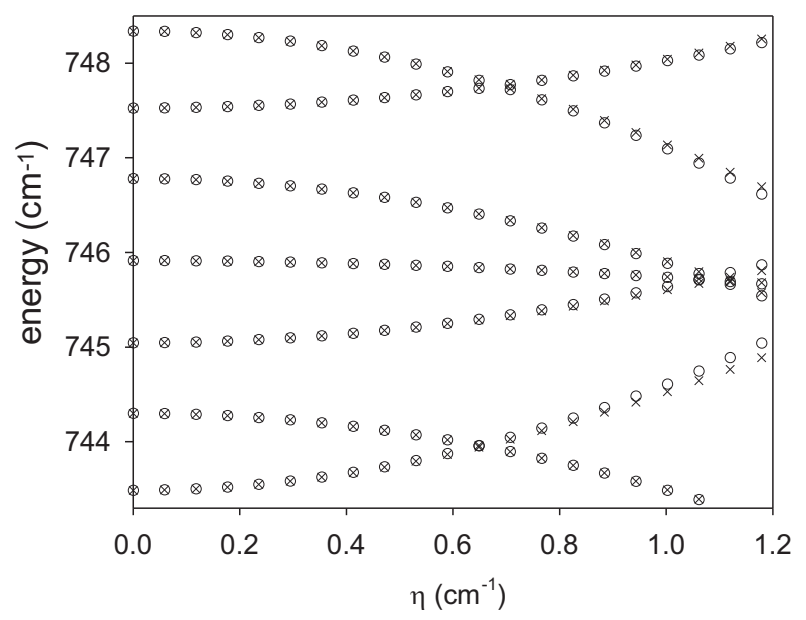

FIG. 2: $\epsilon_{\mu}$ (thin $\left.\mathrm{x}\right)$ and $\epsilon_{k, n}$ (circles) versus $\eta$ for $N=11$.

$\Delta \epsilon=4.44 \times 10^{-5} \mathrm{~cm}^{-1}$ is very small. By contrast, for the eigenstate $\mu=900\left(\epsilon_{900}=1026.10 \mathrm{~cm}^{-1}\right)$ connected to $(k=1, n=80), \Delta \epsilon$ increases to reach $4.32 \times 10^{-3}$ $\mathrm{cm}^{-1}$. Nevertheless, $\Delta \epsilon$ is always smaller than the energy level spacing so that PT remains valid. Note that the curve $\Delta \epsilon$ vs $\epsilon_{\mu}$ behaves almost periodically indicating that the PT accuracy depends on the nature of the unperturbed states. Over a large energy scale, it seems that unperturbed states involving $k=N$ are the less well-corrected.

Although $\Delta \epsilon$ increases with $\chi$, Fig. 2 reveals that PT mimics quite well the $\eta$ dependence of the energy levels, even in the intermediate coupling regime (for $N=11$, $\chi=20 \mathrm{pN}$ yields $\left.\eta=1.18 \mathrm{~cm}^{-1}\right)$. In particular, PT describes the energy level crossing phenomena that affects the dressed states. Due to the coupling $V$, unperturbed states hybridize giving rise to energy shift and splitting characteristic of anti-crossing processes. Consequently, some energy levels repel each other whereas other energy levels get closer. These levels describing exact uncoupled eigenstates, energy level crossing occurs.

At this step, we have verified that $\mathrm{PT}$ yields good estimates of the eigenstates (not drawn). For $\chi=8 \mathrm{pN}$, the main contribution of each eigenstate is collinear to the corresponding unperturbed state whose weight is wellaccounted by PT. Nevertheless, for high energy levels or for larger $\chi$ values, a small discrepancy occurs between numerical calculations and PT, especially for describing the weight of the states $|k \pm 1, n \pm 1\rangle$. This feature arises because these contributions result from first order PT whereas the other contributions are exact up to second order.

The $\eta$ dependence of the phonon energy correction $\delta \Omega_{k}^{(2)}$ is illustrated in Fig. 3. The intensity and the sign of $\delta \Omega_{k}^{(2)}$ depend on the nature of the exciton that accompanies the phonon. An exciton whose energy is above $\omega_{0}$ yields a red-shift of the phonon energy. Moreover, the closer to the band edge the exciton energy is located, the larger $\delta \Omega_{k}^{(2)}$ is. By contrast, an exciton whose energy 


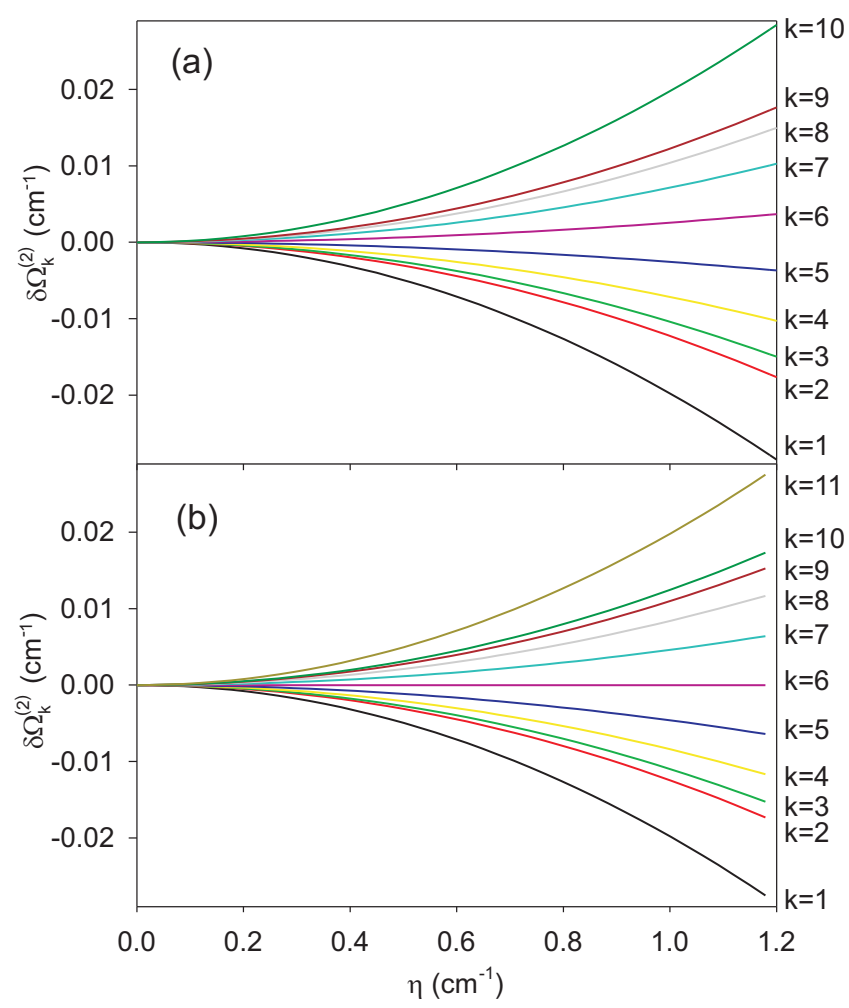

FIG. 3: (Color online) $\eta$ dependence of the phonon energy correction $\delta \Omega_{k}^{(2)}$ for (a) $N=10$ and (b) $N=11$.

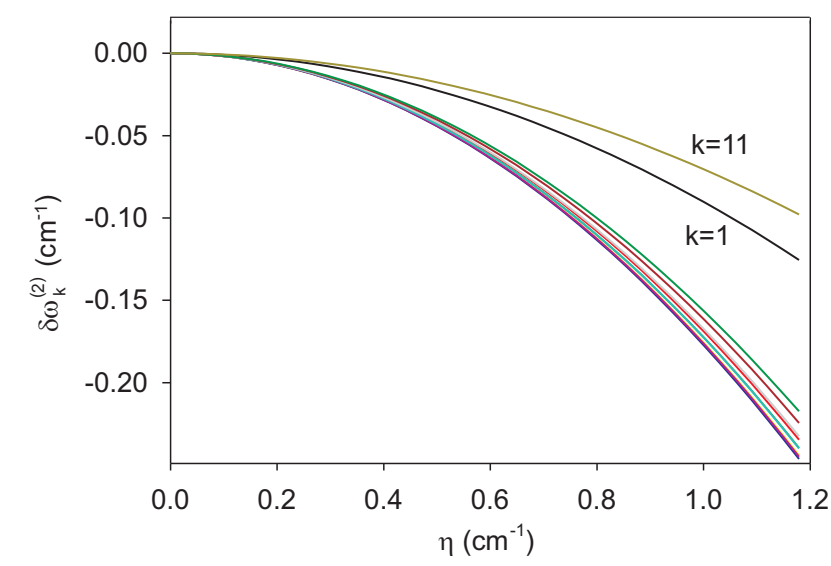

FIG. 4: (Color online) $\eta$ dependence of the exciton energy correction $\delta \omega_{k}^{(2)}$ for $N=11$.

is below $\omega_{0}$ leads to a blue-shift of the phonon energy. As previously, excitonic states near the band edge yield the largest phonon energy shift. A surprising effect takes place for odd lattice sizes. In that case, a phonon clothed by an exciton whose energy is exactly located at the band center is not perturbed. In other words, for $k=L / 2$, one obtains $\delta \Omega_{L / 2}^{(2)}=0 \forall \eta$.

Fig. 4 displays the $\eta$ dependence of the exciton energy correction $\delta \omega_{k}^{(2)}$. Note that $\delta \omega_{k}^{(2)}$ is almost insensitive to the parity of the lattice size so that only the case $N=11$

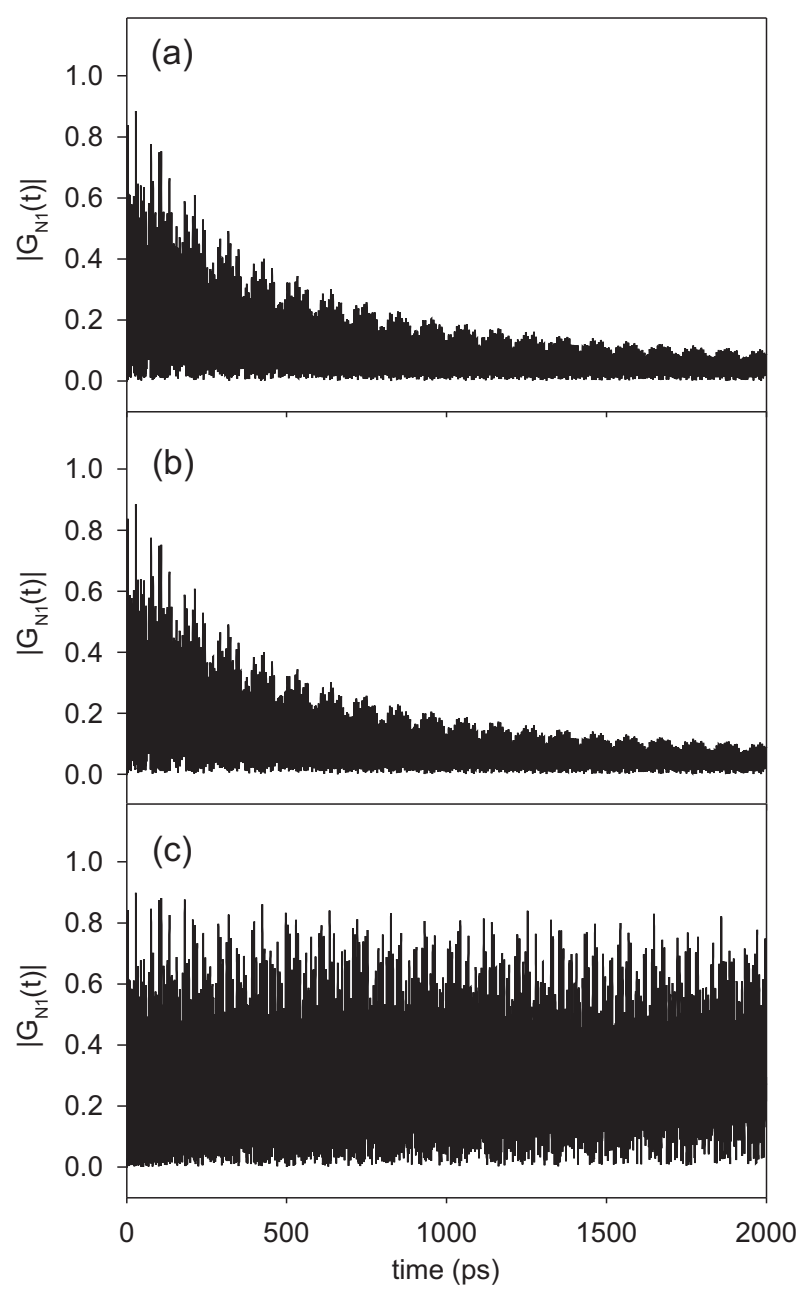

FIG. 5: Excitonic propagator in the local basis for $N=8$, $T=300 \mathrm{~K}$ and $\chi=8 \mathrm{pN}$. (a) Exact calculation, (b) PT and (c) $\mathrm{TCL}_{2}$-GME.

is reported. The figure shows that the dressing by virtual phonons is responsible for a red-shift of the exciton energy so that $\delta \omega_{k}^{(2)}$ decreases as $\eta$ increases. In absolute value, it is typically two orders of magnitude larger than $\left|\delta \Omega_{k}^{(2)}\right|$. Fig. 4 reveals that the sensitivity of the states $k=2, \ldots, N-1$ to the dressing effect is almost $k$ independent. In other words, these states experience a similar energy shift whose value is typically of about $-2 E_{B} / L$. By contrast, the states located at the band edges $k=1$ and $k=N$ appear weakly coupled with the phonons. They exhibit almost the same energy shift whose value, of about $-E_{B} / L$, is typically two times smaller than the energy correction of the other states.

To characterize the coherence transfer along the lattice, let us consider the time evolution of the effective exciton propagator in the local basis (Eq.(5)). Special attention is paid to describe the ability of the system to transfer a quantum state between the two lattice sides, $x_{1}=1$ and $x_{2}=N$. Such information is encoded in the propagator $\left|G_{N 1}(t)\right|$ whose time evolution is shown 
in Fig. 5 for $N=8, T=300 \mathrm{~K}$ and $\chi=8 \mathrm{pN}$. Note that Fig. 5 displays results provided by exact calculations (Fig. 5a), PT (Fig. 5b) and $\mathrm{TCL}_{2}$-GME method (Fig. 5c $)^{48}$.

Although it is not really clear over the time scale shown in Fig. 5, we have verified that both $\mathrm{PT}$ and $\mathrm{TCL}_{2^{-}}$ GME yield a quite good estimate of the propagator in the short time limit $(t<100 \mathrm{ps})$. However, as time increases, $\mathrm{TCL}_{2}$-GME fails in reproducing the time evolution of the propagator and suggests, wrongly, that an efficient coherence transfer occurs over a rather long time scale. This is no longer the case for PT that describes accurately the time evolution of the transmitted coherence, even in the long time limit. According to Figs. 5a and $5 \mathrm{~b}$, the exciton propagator behaves as follows. Initially equal to zero, $\left|G_{N 1}(t)\right|$ reaches a first maximum that characterizes a direct exciton transfer between the two lattice sides. Then, due to the confinement, $\left|G_{N 1}(t)\right|$ does not vanish but it exhibits peaks which are almost periodically distributed. These peaks define quantum recurrences that occur at either classical periods, revival times or super-revival times ${ }^{47,74}$. Nevertheless, as time increases, the intensity of the quantum recurrences decreases indicating that a rather slow quantum decoherence takes place. Note that the coherence decays rather algebraically than exponentially. For instance, around $100 \mathrm{ps}$, the maximum value of the propagator is about 0.75 . It becomes typically of about 0.2 around $1000 \mathrm{ps}$ and reduces to 0.1 near 2000 ps. In other words, the transmitted coherence exhibits small amplitude fluctuations around a mean value which apparently tends to zero as time reaches $2000 \mathrm{ps}$. We have verified that such a behavior is characteristic of a lattice whose size is an even number.

As shown in Fig. 6, similar results are observed for $N=9$. Note that the $\mathrm{TCL}_{2}$-GME method is not reported in Fig. 6 since it gives a time evolution quite similar to that displayed in Fig. 5c. Both PT and $\mathrm{TCL}_{2}$-GME yield accurate results in the short time limit and they reveal the occurrence of peaks regularly distributed. Nevertheless, as time increases, $\mathrm{TCL}_{2}-\mathrm{GME}$ breaks down and only $\mathrm{PT}$ provides results in good agreements with the exact calculations. The transmitted coherence still displays quantum recurrences whose intensity slightly decreases as time increases. However, it is as if the transmitted coherence converged to a non vanishing value of about 0.17 by exhibiting high frequency oscillations whose amplitude is about 0.8. In fact this feature is rather general and it occurs for an odd lattice size.

At this step, let us mention that long time simulations have been carried out over a time scale equal to $20000 \mathrm{ps}$ (not drawn). Coherence revivals have been observed indicating that the transmitted coherence does not vanish whatever the parity of the lattice size. These features will be discussed later. Moreover, for larger $\chi$ values, we have observed that PT remains quite good. For instance, for $\chi=16 \mathrm{pN}, \mathrm{PT}$ captures the main part of the propaga-

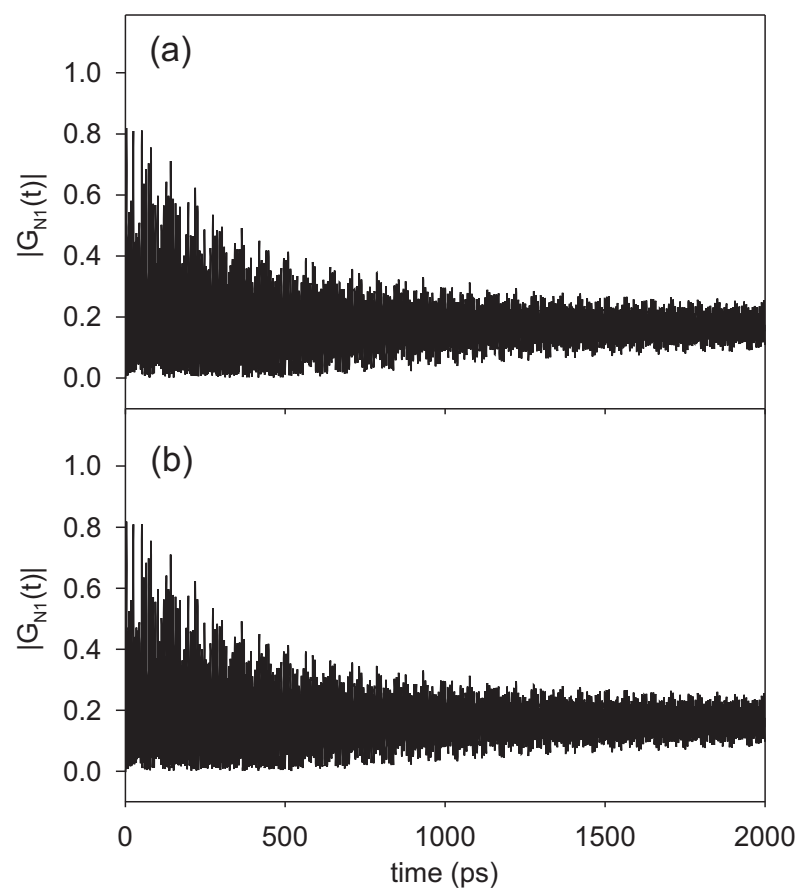

FIG. 6: Excitonic propagator in the local basis for $N=9$, $T=300 \mathrm{~K}$ and $\chi=8 \mathrm{pN}$. (a) Exact calculation and (b) PT.

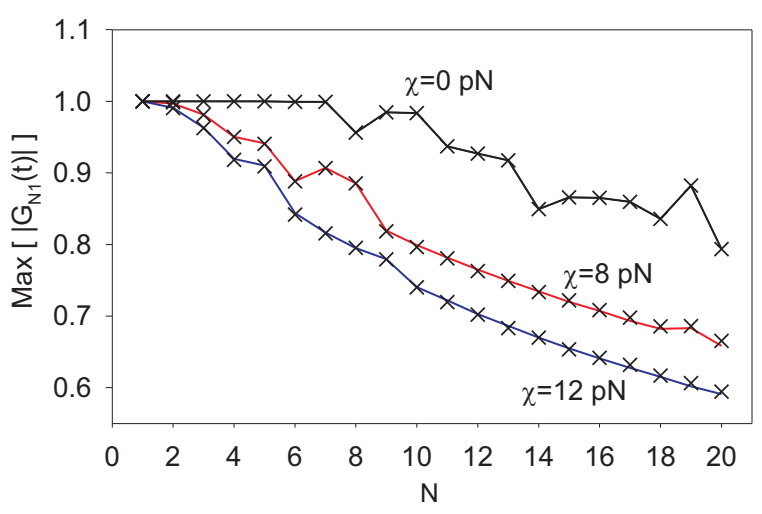

FIG. 7: (Color online) Maximum value of the transmitted coherence over $1000 \mathrm{ps}$ versus lattice size for $\chi=0,8$ and 12 pN. Exact calculations (full lines) and PT (x symbols).

tor whereas $\mathrm{TCL}_{2}$-GME yields an unphysical exponential growth.

Information about the optimized coherence transfer is displayed in Fig. 7 for $T=300 \mathrm{~K}$. For a lattice containing $N$ sites, Fig. 7 shows the maximum value of the transmitted coherence, i.e. $t_{N}=\operatorname{Max}\left[\left|G_{N 1}(t)\right|\right]$, which occurs over an observation time scale equal to 1000 ps. For $\chi=0 \mathrm{pN}, t_{N}=1$ provided that $N \leq 7$. Then, it slightly decreases with $N$ giving rise to a high fidelity transfer. Note that a linear regression yields $t_{N} \approx 1-0.013(N-7)$ for $N \geq 7$. In fact, when $\chi=0 \mathrm{pN}$, the coherence properties are governed by the exciton Hamiltonian that favors quantum recurrences. Consequently, the maximum of $\left|G_{N 1}(t)\right|$ occurs after the exciton has experienced several 
reflections. For instance, for $N=16, t_{N}=0.86$ is very close to unity, the maximum taking place at $t=852.2$ ps. When the exciton-phonon interaction is turned on, the intensity of the quantum recurrences is reduced (see Figs. 5 and 6 ) so that $t_{N}$ decreases. For $\chi=8 \mathrm{pN}$, it typically scales $t_{N} \approx 1-0.019(N-1)$ whereas for $\chi=12$ $\mathrm{pN}$, it behaves as $t_{N} \approx 1-0.022(N-1)$. Note that a critical size $N_{c} \approx 8$ discriminates between two regimes. When $N<N_{c}$, the maximum of $\left|G_{N 1}(t)\right|$ takes place at either a revival time or a super-revival time for which the exciton has experienced many reflections. By contrast, for $N>N_{c}$, the most probable event giving rise to the maximum of $\left|G_{N 1}(t)\right|$ is a direct transfer between the lattice sides.

Let us now study the time evolution of the coherences expressed in the excitonic eigenbasis. To proceed, we shall restrict our attention to the diagonal elements $G_{k k}(t)$ of the effective propagator. These elements define the coherence of quantum states involving the vacuum and the one-exciton eigenstates. To simplify, $G_{k k}(t)$ will be called the coherence of the state $|k\rangle$. Such a restriction is valid because the propagator is diagonally dominant. More precisely, our numerical study reveals that $G_{k_{2} k_{1}}(t) \neq 0$ for $\left|k_{2}-k_{1}\right|=0,2,4, \ldots$, only. As predicted by PT (Eq.(B1)), diagonal terms are the most intense since they involve the contribution of the eigenstates collinear to the unperturbed states. By contrast, non diagonal terms $\left|k_{2}-k_{1}\right|=2$ remain small since they are proportional to the square of the coupling. Finally, PT does not account on non diagonal terms $\left|k_{2}-k_{1}\right|=4,6, \ldots$ that involve high order contributions of the coupling. Fortunately, these latter terms almost vanish in the weak coupling limit.

For an even lattice size $(N=8),\left|G_{k k}(t)\right|$ is displayed in Fig. 8 for $T=300 K$ and $\chi=8 \mathrm{pN}$. The figure clearly shows the agreement between exact calculations (Fig. 8a) and PT (Fig. 8b). In the short time limit $(t<50-100 \mathrm{ps})$, it reveals that the coherences exhibit high frequency small amplitude fluctuations just below their initial value equal to unity. Then, as time increases, the coherences decrease indicating that quantum decoherence takes place over 2000 ps. Note that this decay is rather algebraic than exponential. Each coherence follows a decaying function that supports a high frequency small amplitude modulation. However, such a behavior depends on the nature of the excitonic state. First, the coherences evolve in pairs so that $\left|G_{k k}(t)\right|$ typically behaves as $\left|G_{L-k L-k}(t)\right|$. Second, the coherence of states close to the band edges decays faster than the coherence of states located near the band center. Finally, the modulation amplitude is more pronounced for states located near the band center.

Let us mention that simulations carried out at different temperatures have revealed that the temperature enhances quantum decoherence. The larger the temperature is, the faster the coherences decay. In addition, as the temperature increases, the modulation amplitude increases. Note that we have verified that the $\mathrm{TCL}_{2}-$

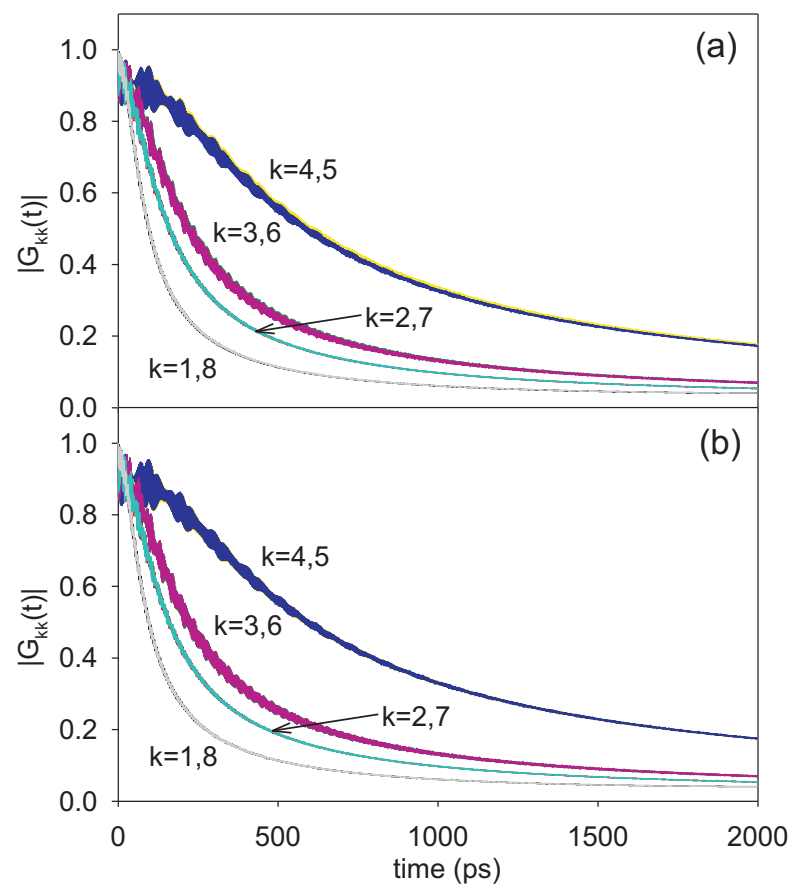

FIG. 8: (Color online) Time evolution of $\left|G_{k k}(t)\right|$ for $N=8$, $T=300 K$ and $\chi=8$ pN. (a) Exact calculations and (b) PT.

GME method provides quite good results in the short time limit $(t<100 \mathrm{ps})$. However, as time increases, it rapidly breaks down and it suggests, wrongly, that the coherences survive over 2000 ps.

For $N=9,\left|G_{k k}(t)\right|$ is shown in Fig. 9 for $T=300 K$ and $\chi=8 \mathrm{pN}$. As previously, a very good agreement is observed between exact calculations (Fig. 9a) and PT (Fig. 9b). Basically, the coherences behave as in Fig. 8. They first exhibit fast oscillations just below their initial value. Then, they follow a decaying function that supports a high frequency small amplitude modulation. Note that the modulation amplitude appears larger, especially for states near the band center. However, a surprising effect occurs that was not observed for an even lattice size. Indeed, the coherence of the state exactly located at the band center $(k=L / 2)$ evolves differently. In the short time limit, it slightly decreases from unity by exhibiting high frequency damped oscillations. As time increases, the oscillations disappear and the coherence converges to a finite value rather close to unity. In other words, the coherence survives over a very long time scale in spite of the coupling with the phonons at high temperature. Note that the influence of the temperature is twofold. First, as the temperature increases, the constant value reached by the coherence decreases. For instance, for $T=100 \mathrm{~K}$ it is equal to 0.96 and it reduces to 0.90 for $T=300 \mathrm{~K}$. Then, the amplitude of the damped oscillations increases with the temperature. Nevertheless, the larger the temperature is, the faster the modulation disappears.

According to the previous results, it would seem that quantum decoherence arises for states $k \neq L / 2$. However, 


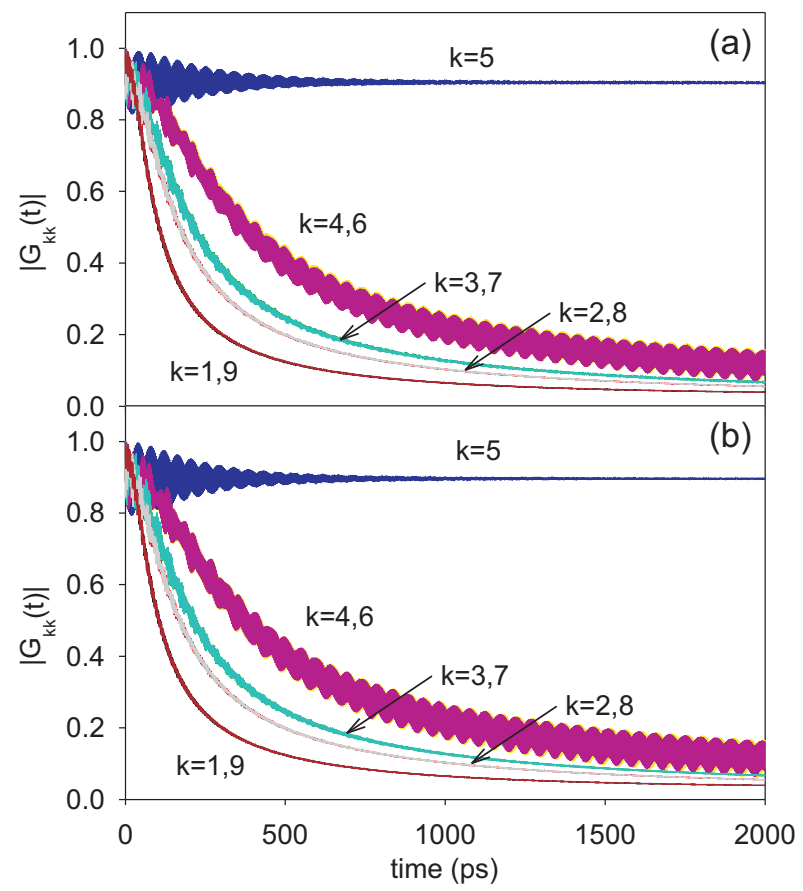

FIG. 9: (Color online) Time evolution of $\left|G_{k k}(t)\right|$ for $N=9$, $T=300 K$ and $\chi=8 \mathrm{pN}$. (a) Exact calculations and (b) PT.

this is a deceptive appearance as illustrated in Fig. 10 for $N=9$. Indeed, for $k \neq L / 2$, each coherence decreases from unity and it finally reaches a minimum value almost $k$ independent. Such a behavior is characterized by the so-called decoherence time $T_{k}^{d}$ for which $\left|G_{k k}\left(T_{k}^{d}\right)\right|=0.5$. However, as time increases, the coherence increases again until it recurs at a specific revival time $T_{k}^{r}$. This scenario continues giving rise to a periodic evolution of the coherence. Its dynamics is thus governed by both the decoherence time and the revival time, whose values strongly depend on the excitonic eigenstates. The closer to the band edges the states are located, the shorter $T_{k}^{d}$ and $T_{k}^{r}$ are. For instance, one obtains $T_{1}^{d}=T_{9}^{d} \approx 110 \mathrm{ps}$ and $T_{1}^{r}=T_{9}^{r} \approx 5300$ ps whereas $T_{2}^{d}=T_{8}^{d} \approx 170 \mathrm{ps}$ and $T_{2}^{r}=T_{8}^{r} \approx 8700 \mathrm{ps}$. The other coherences also recur but for revival times longer than the time scale reported in the figure. As mentioned previously, the decoherence time decreases with the temperature. By contrast, the revival time is typically temperature independent. Nevertheless, the temperature modifies the coherence revivals whose intensity decreases as the temperature increases.

As shown in Fig. 10, PT mimics quite well the exact calculations over a very long time scale. Nevertheless, a small discrepancy occurs between exact calculations and $\mathrm{PT}$ for describing the coherence of the state $k=L / 2$. Indeed, according to PT, this coherence converges whereas Fig. 10a reveals that it slightly decreases as time increases. In fact, this coherence exhibits both quantum decoherence and coherence revival but over a very long time scale. To illustrate this feature, Fig. 11 displays the time evolution of this coherence for $\chi=12 \mathrm{pN}$, i.e.

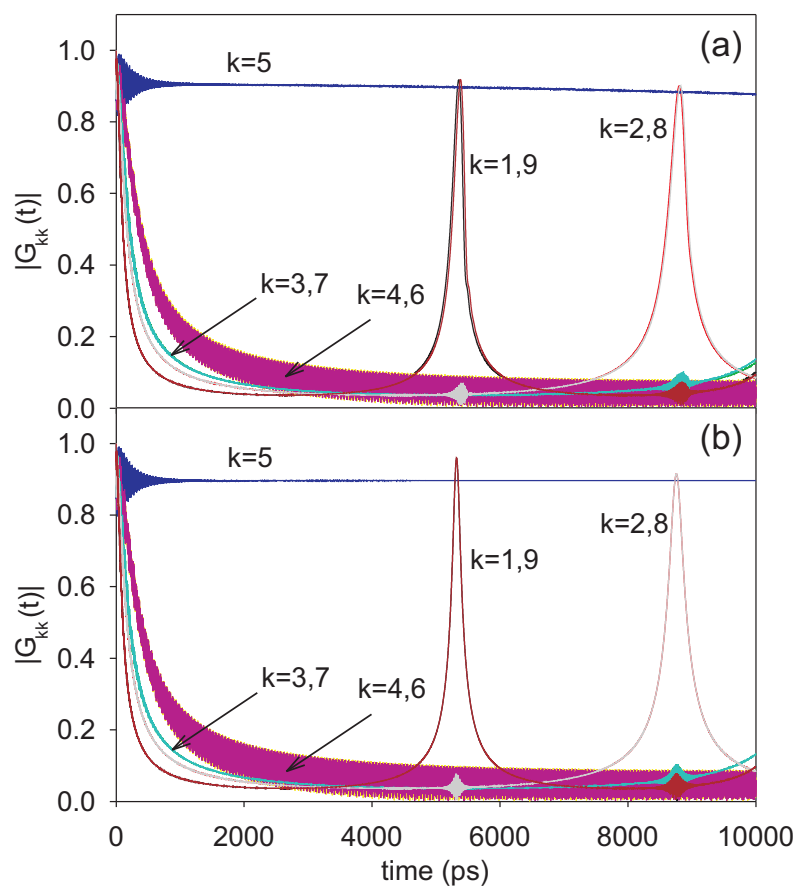

FIG. 10: (Color online) Long time evolution of $\left|G_{k k}(t)\right|$ for $N=9, T=300 K$ and $\chi=8 \mathrm{pN}$. (a) Exact calculations and (b) PT.

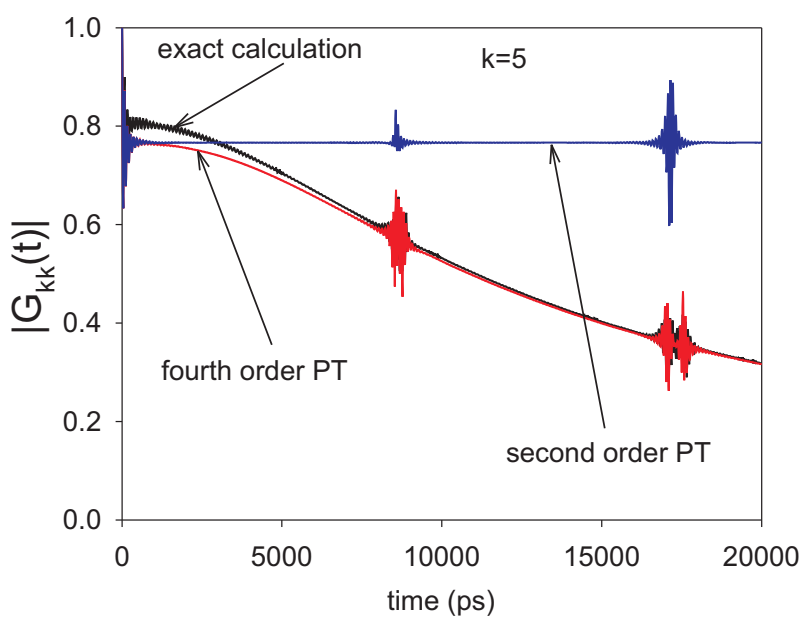

FIG. 11: (Color online) Long time evolution of the excitonic coherences $\left|G_{k k}(t)\right|$ for $k=L / 2, N=9, T=300 K$ and $\chi=12 \mathrm{pN}$.

a coupling value for which $T_{L / 2}^{d}$ is shortened to $11050 \mathrm{ps}$. In that case, quantum decoherence is clearly evidenced, as well as PT breakdown. Note that the other coherences remain quite well-described by PT (not drawn). To overcome this difficulty, a fourth order PT has been applied to improve the energy correction of the unperturbed states (Appendix C). Doing so, we have verified that the fourth order PT only slightly modifies the time evolution of the coherence of the states $k \neq L / 2$. By contrast, it strongly affects the time evolution of the coherence of the state 
$k=L / 2$ whose long time behavior now resembles the time evolution provided by exact calculations.

To conclude this section, let us mention that a numerical simulation of the phonon reduced density matrix $r(t)=\operatorname{Tr}_{A}[\rho(t)]$ has been carried out. The main idea was to clarify if the presence of an exciton strongly affects the phonon properties in the weak coupling limit. Therefore, initially in thermal equilibrium, we have observed that the phonon statistical state is only slightly modified. Indeed, the populations $r_{n n}(t)$ of the phonon number states $|n\rangle$ are no longer constant. They exhibit small amplitude fluctuations around their equilibrium value. In addition, phonon coherences $r_{n n^{\prime}}(t)$ switch on. When the exciton occupies a local state at $t=0$, coherences with $\left|n-n^{\prime}\right|=1,2,3 \ldots$ turn on. By contrast, phonon coherences with $\left|n-n^{\prime}\right|=2,4,6 \ldots$ occur when the exciton is initially created in an eigenstate. Fortunately, the phonon coherences are very small so that it is as if the phonons still remained in a quasi-equilibrium with a slightly fluctuating temperature.

\section{DISCUSSION}

The numerical results show that PT is a powerful tool for describing the exciton-phonon system in a confined environment. Within the nonadiabatic weak coupling limit, it yields a very good estimate of the spectral properties of the system over a rather large energy scale. The behavior of the eigenenergies is encoded into two parameters, namely the exciton energy correction $\delta \omega_{k}^{(2)}$ and the phonon energy correction $\delta \Omega_{k}^{(2)}$. These parameters account for both the dressing of the exciton by a virtual phonon cloud and the dressing of each phonon by excitonic virtual transitions. Furthermore, PT is particularly suitable for describing the dynamics of the excitonic coherences over short, intermediate and long time scales. In that sense, it is more accurate than second order master equation approaches that quite well mimic the dynamics in the short time limit but break down over intermediate and long time scales.

Our results reveal that the evolution of the excitonic coherences is governed by several time scales. In the short time limit $(t<100 \mathrm{ps}$ for amide-I exciton), the coherences in the excitonic eigenbasis exhibit high frequency small amplitude fluctuations just below their initial value equal to unity. It is as if the coherences survived in spite of the exciton-phonon coupling at finite temperature. In the local basis, the transmitted coherence evolves almost periodically and it shows peaks that characterize quantum recurrences. As time increases, quantum decoherence takes place over a time scale specified by the socalled decoherence time. The coherence of each eigenstate slowly decreases rather algebraically than exponentially. Similarly, quantum decoherence favors an algebraic decay of the transmitted coherence and the quantum recurrences decrease as time increases. Nevertheless, the coherences do not vanish, neither in the local basis nor in the eigenbasis. Quantum decoherence is only transient and the coherences in the eigenbasis recur at specific revival times. The main observation is that both decoherence times and revival times strongly depend on the nature of the excitonic states. The closer to the band center the states are located, the longer the decoherence times and the revival times are. In particular, for odd lattice sizes, they become extremely long for the state exactly located at the band center.

To interpret the time evolution of the coherences, let us introduce two simplifying approximations. First, the effective propagator being diagonally dominant in the excitonic eigenbasis, a diagonal element $G_{k k}(t)$ characterizes a coherence $\sigma_{k \oslash}(t)$, i.e. $\sigma_{k \oslash}(t) \approx G_{k k}(t) \sigma_{k \oslash}(0)$. Then, in the weak coupling limit, the eigenstates being almost collinear to the unperturbed states, one assumes $\left|\Psi_{k, n}\right\rangle \approx|k, n\rangle$. Consequently Eq.(10) yields

$$
G_{k k}(t) \approx e^{-i \hat{\omega}_{k} t} \sum_{n=0}^{\infty} P_{n} e^{i n\left(\Omega-\hat{\Omega}_{k}\right) t}
$$

The physics provided by Eq.(12) can be understood as follows. At $t=0$, the system is prepared in a factorized state $|\Psi(0)\rangle=\left[c_{0}|\oslash\rangle+c_{1}|k\rangle\right] \otimes|n\rangle$. This state describes $n$ phonons accompanied by an exciton in a superimposed state, phonons and exciton being independent. As time increases, this state evolves in an entangled exciton-phonon state as

$$
|\Psi(0)\rangle \approx c_{0}|\oslash\rangle \otimes\left|\phi_{n}^{(\oslash)}(t)\right\rangle+c_{1} e^{-i \hat{\omega}_{k} t}|k\rangle \otimes\left|\phi_{n}^{(k)}(t)\right\rangle
$$

where $\left|\phi_{n}^{(\oslash)}(t)\right\rangle=\exp (-i n \Omega t)|n\rangle$ is the state of $n$ free phonons whereas $\left|\phi_{n}^{(k)}(t)\right\rangle=\exp \left(-i n \hat{\Omega}_{k} t\right)|n\rangle$ describes the evolution of $n$ phonons dressed by the exciton. After performing a trace over the phonon degrees of freedom, the coherence scales as $G_{k k}(t) \approx$ $\exp \left(-i \hat{\omega}_{k} t\right)\left\langle\phi_{n}^{(\oslash)}(t) \mid \phi_{n}^{(k)}(t)\right\rangle$. The phase factor characterizes the evolution of the exciton dressed by a virtual phonon cloud. It does not significantly modify the coherence dynamics. By contrast, the so-called decoherence factor $^{36,37}\left\langle\phi_{n}^{(\oslash)}(t) \mid \phi_{n}^{(k)}(t)\right\rangle$ measures the ability of the phonons to evolve freely in spite of their interaction with the exciton. When the phonons are initially in a pure state, the decoherence factor reduces to a phase factor $\exp \left(i n\left(\Omega-\hat{\Omega}_{k}\right) t\right)$ that involves the energy difference between free and dressed phonons. This latter phase factor does not affect the excitonic coherence. Unfortunately, at finite temperature, the phonons are described by a statistical mixture of number states. As shown in Eq.(12), the coherence is thus proportional to the average value of the decoherence factor. Performing the average, its modulus is written as

$$
\left|G_{k k}(t)\right| \approx \frac{1}{\sqrt{1+4 \Delta \bar{n}^{2} \sin ^{2}\left(\frac{1}{2} \delta \Omega_{k}^{(2)} t\right)}}
$$

where $\Delta \bar{n}^{2}=\bar{n}(\bar{n}+1)$ measures the thermal fluctuations of the phonon number. In spite of its simple appearance, 
Eq.(14) allows us to recover the main features observed in our numerical analysis.

First, due to the symmetry of the phonon energy correction, i.e. $\delta \Omega_{k}^{(2)}=-\delta \Omega_{L-k}^{(2)}$ (Fig. 3), the coherences evolve in pairs. One obtains $\left|G_{k k}(t)\right|=\left|G_{L-k L-k}(t)\right|$, in good agreements with the results displayed in Figs. 8, 9 and 10 .

Second, Eq.(14) reveals that the average over the initial phonon number leads to quantum decoherence. Indeed, when $\delta \Omega_{k}^{(2)} t \ll 1$, the coherence, initially equal to unity, slowly decreases as time increases. It does not decrease in an exponential way. Instead, it exhibits an algebraic decay and scales as $\left|G_{k k}(t)\right| \approx\left[1+\left(\Delta \bar{n} \delta \Omega_{k}^{(2)} t\right)^{2}\right]^{-1 / 2}$. This decay is characterized by a decoherence time $T_{k}^{d}$ defined as $\left(\left|G_{k k}\left(T_{k}^{d}\right)\right|=1 / 2\right)$,

$$
T_{k}^{d}=\frac{\sqrt{3}}{\Delta \bar{n}\left|\delta \Omega_{k}^{(2)}\right|}
$$

Since $\Delta \bar{n}$ scales as $T, \operatorname{Eq}(15)$ shows that the temperature enhances quantum decoherence. Moreover, $T_{k}^{d}$ is inversely proportional to the square of the coupling through its dependence with respect to the phonon energy correction (see Eq.(8)). From this dependence, we recover that the closer to the band edges the excitonic state is located, the shorter $T_{k}^{d}$ is. For instance, with the parameters used in Fig. 10, Eq.(15) yields $T_{1}^{d}=T_{9}^{d}=106.65$ ps and $T_{2}^{d}=T_{8}^{d}=175.52 \mathrm{ps}$, in good agreements with the numerical estimates. As observed in Fig. 9, Eq.(15) reveals that the coherence of the excitonic state exactly located at the band center $(k=L / 2)$ survives over an infinite time scale. This feature occurs for an odd lattice size only because $\delta \Omega_{L / 2}^{(2)}$ vanishes due to the symmetry of the exciton energy spectrum. Indeed, during both phonon absorption and phonon stimulated emission, the virtual excitonic transition form $|k\rangle$ to $|k+1\rangle$ is counterbalanced by the transition form $|k\rangle$ to $|k-1\rangle$. When $k=L / 2$, both contributions exactly compensate resulting in a zero phonon energy shift so that $T_{L / 2}^{d} \rightarrow \infty$. Of course, such a behavior results from a second order $\mathrm{PT}$ whereas exact calculations reveal that $T_{L / 2}^{d}$ is very long but remains finite. To account on this feature, a fourth order PT has been realized (see Appendix C). In that case, fourth order corrections $\delta \Omega_{k}^{(4)}$ remain negligible when compared with $\delta \Omega_{k}^{(2)}$, excepted for $k=L / 2$ since $\delta \Omega_{L / 2}^{(2)}=0$. The corresponding decoherence time is about $T_{L / 2}^{d}=\sqrt{3} / \Delta \bar{n}\left|\delta \Omega_{L / 2}^{(4)}\right|$. With the parameters used in Fig. 11, one obtains $T_{L / 2}^{d}=11816$ ps in quite good agreements with the numerical results.

At this step, let us mention that the so-called decoherence rate $\Gamma_{k}^{d}=\Delta \bar{n} \delta\left|\Omega_{k}^{(2)}\right| / \sqrt{3}$ depends quadratically on the coupling strength. However, its temperature dependence is encoded in the thermal fluctuations of the phonon number. In that sense, it differs from the standard expression of the dephasing rate that characterizes the decoherence of an open system coupled with a reservoir of harmonic oscillators ${ }^{2}$. In that case, GME methods show that the temperature dependence of the rate originates in its dependence with respect to the average phonon number $\bar{n}$. At high temperature, both approaches yield a similar temperature dependence since $\Delta \bar{n} \approx \bar{n} \approx k_{B} T / \Omega$. This is no longer the case at low temperature since $\Delta \bar{n} / \bar{n}=\exp (\beta \Omega / 2)$. Inserting Eq.(8) into Eq.(15) for $k=1$ (or $k=N$ ) reveals that the largest decoherence rate scales as $\Gamma_{1}^{d} \approx 13.85 \Phi E_{B} k_{B} T /\left(\Omega_{c}^{2} L\right)$ at high temperature. It decreases as $L$ increases provided that $L<L^{*}$. This latter condition ensures that $\Gamma_{1}^{d}$ remains smaller than the smallest excitonic Bohr frequency $\Delta \omega \approx 3 \Phi \pi^{2} / L^{2}$.

Finally, Eq.(14) shows that the coherence is a periodic function of time so that quantum decoherence is only transient. The coherence does not vanish and it reaches a minimum at time $t_{k}=\pi /\left|\delta \Omega_{k}^{(2)}\right|$. This minimum, equal to $1 / \sqrt{1+4 \Delta \bar{n}^{2}}$, only depends on the thermal fluctuations of the phonon number. It thus decreases as the temperature increases. As time increases, the coherence increases again and it finally recurs at a revival time $T_{k}^{r}=2 \pi /\left|\delta \Omega_{k}^{(2)}\right|$. The revival time is temperature independent and it only depends on the invert of the phonon energy correction. As a result, the closer to the band edges the excitonic state is located, the shorter $T_{k}^{r}$ is. For instance, with the parameters used in Fig. 10, one obtains $T_{1}^{r}=T_{9}^{r}=5322 \mathrm{ps}$ and $T_{2}^{r}=T_{8}^{r}=8758$ ps, in good agreements with the numerical estimates. As for the decoherence time, second order PT predicts an infinite revival time for the coherence of the state $k=L / 2$. However, within fourth order PT (Appendix C), $\delta \Omega_{L / 2}^{(4)}$ does no longer vanish. With the parameters used in Figs. 10 and 11 , one obtains $T_{L / 2}^{r} \approx 2.98 \times 10^{6}$ ps and $T_{L / 2}^{r} \approx 5.89 \times 10^{5} \mathrm{ps}$, for $\chi=8 \mathrm{pN}$ and $\chi=12$ $\mathrm{pN}$, respectively. Note that Eq.(14) describes exact revivals for which the coherence reaches unity. This feature results form the assumption that the eigenstates reduce to the unperturbed states. However, by using Eq.(B1), the temperature effect is taken into account so that the maximum of the coherence decreases as the temperature increases.

In the local basis, the transmitted coherence is a superimposition of the coherences in the eigenbasis as

$$
G_{N 1}(t) \approx-\frac{2}{L} \sum_{k=1}^{N}(-1)^{k} \sin ^{2}\left(\frac{k \pi}{L}\right) G_{k k}(t)
$$

In the short time limit, $G_{N 1}(t)$ scales as the propagator of a bare exciton. It measures the ability of the system to copy the initial state on the side site $x=N$. The initial excitation on site $x=1$ yields the emission of a wave packet that propagates with a group velocity $v \approx 2 \Phi$. After a time $\tau_{0} \approx L / v$, the wave packet reaches the site $x=N$ and a peak occurs in $G_{N 1}(t)$. As observed in Figs. 5 and 6 , such a mechanism takes place almost periodically and some peaks in $G_{N 1}(t)$ define recurrences that 
occur according to the so-called classical period $2 \tau_{0}{ }^{47,74}$. They describe situations in which the exciton quantum state partially resembles the copy of the initial state but localized on the site $x=N$. Moreover, the discrete nature of the exciton energy spectrum provides both revival times and super-revival times for which the reappearance of the wave packet on the site $x=N$ yields quantum states that strongly resemble the initial state ${ }^{47,74}$. Unfortunately, as time increases, quantum decoherence takes place and the intensity of these quantum recurrences decreases. For an even lattice size, each coherence $G_{k k}(t)$ decreases as time increases so that $\left|G_{N 1}(t)\right|$ slowly decays. It finally exhibits small amplitude fluctuations close to zero, as displayed in Fig. 5. By contrast, for an odd lattice size, the coherence of the state $k=L / 2$ survives so that $\left|G_{N 1}(t)\right|$ shows small amplitude oscillations around an average value of about $2 / L$, as illustrated in Fig. 6. Note that over longer time scales, coherence revivals in $G_{k k}(t)$ give rise to quantum recurrences in the transmitted coherence. However, these recurrences remain weak because different coherences $G_{k k}(t)$ are characterized by different revival times. It is unlikely that coherences $G_{k k}(t)$ will recur simultaneously to provide a strong recurrence in $G_{N 1}(t)$.

To conclude, we would like to point out that, in addition to its academic interest, the present work reveals interesting features that may be applied to quantum information processing in condensed matter. Indeed, it has been shown that the confinement softens the quantum decoherence experienced by an exciton coupled with a phonon bath, even at high temperature. This softening is particular important, at least, for two main reasons. First, the confinement enhances the ability of the system to promote high fidelity quantum state transfer. Over an observation time scale of about few times the decoherence time, the maximum value of the transmitted coherence decreases almost linearly with the lattice size. In short lattices, the exciton realizes many reflections before experiencing quantum decoherence so that the optimized transmitted coherence occurs at either a revival time or a super-revival time. By contrast, in longer lattices, a direct quantum state transfer takes place between the lattice sides. This feature is fully different from the behavior observed in an infinite lattice in which dephasinglimited coherent motion yields a strong localization of the transmitted coherence that decreases exponentially with distance ${ }^{44}$. Second, the excitonic state exactly located at the band center is almost insensitive to the phonon bath. Consequently, a superimposition involving the vacuum and this particular one-exciton state is able to keep its coherent nature over an extremely long time scale. Of course, this effect arises provided that the weak coupling limit is reached. However, our results have shown that the decoherence time remains very long even in the intermediate coupling regime. Consequently, at high temperature, such a superimposition can be viewed as an ideal quantum bit insensitive to quantum decoherence, the public enemy number one in quantum computing.

\section{CONCLUSION}

In the present paper, the properties of the excitonphonon system in a confined environment has been revisited within PT. Special attention has been paid for describing the time evolution of the excitonic coherences. To proceed, the system involving an exciton dressed by a single phonon mode has been considered. Due to its simplicity, it was solved exactly so that the PT performance has been checked. Within the nonadiabatic weak coupling limit, it has been shown that PT is a powerful tool for describing the spectral properties of the system over a rather large energy scale. Moreover, it is particularly suitable for characterizing the coherence dynamics over short, intermediate and long time scales. It is thus more accurate than second order master equation approaches that rapidly break down.

We have shown that several time scales govern the dynamics of the coherences. In the short time limit, the coherences behave as if the exciton was insensitive to the phonon bath. Then, quantum decoherence takes place over a time scale specified by the decoherence time. However, quantum decoherence is only transient so that the coherences do not vanish in the long time limit. Instead, they recur periodically at specific revival times. Both decoherence times and revival times strongly depend on the nature of the excitonic states. In particular, for odd lattice sizes, it has been shown that the coherence of the state exactly located at the band center survives over an extremely long time scale. In that context, it has been pointed out that confinement-induced decoherence softening favors high fidelity quantum state transfer. Furthermore, it allows to encode the information on a quantum bit almost insensitive to quantum decoherence, even at high temperature.

Finally, since the present approach has revealed the powerfulness of PT, it will be generalized for describing an exciton coupled with all the phonon modes in a confined environment and at finite temperature. 


\section{APPENDIX A: EIGENSTATES OF THE FULL HAMILTONIAN $H$}

According to the standard second order PT, the eigenstates of the full Hamiltonian $H$ are expressed as

$$
\begin{aligned}
\left|\Psi_{k, n}\right\rangle & =|k, n\rangle \\
& +\sqrt{n+1}\left[\mu_{k}^{+}|k+1, n+1\rangle+\nu_{k}^{+}|k-1, n+1\rangle\right] \\
& +\sqrt{n}\left[\mu_{k}^{-}|k+1, n-1\rangle+\nu_{k}^{-}|k-1, n-1\rangle\right] \\
& -\frac{1}{2}\left[(n+1)\left(\mu_{k}^{+2}+\nu_{k}^{+2}\right)+n\left(\mu_{k}^{-2}+\nu_{k}^{-2}\right)\right]|k, n\rangle \\
& +\sqrt{(n+1)(n+2)} \mu_{k}^{+} u_{k}^{+}|k+2, n+2\rangle \\
& +\sqrt{(n+1)(n+2)} \nu_{k}^{+} v_{k}^{+}|k-2, n+2\rangle \\
& +\sqrt{n(n-1)} \mu_{k}^{-} u_{k}^{-}|k+2, n-2\rangle \\
& +\sqrt{n(n-1)} \nu_{k}^{-} v_{k}^{-}|k-2, n-2\rangle \\
& +u_{k}^{0}\left[(n+1) \mu_{k}^{+}+n \mu_{k}^{-}\right]|k+2, n\rangle \\
& +v_{k}^{0}\left[(n+1) \nu_{k}^{+}+n \nu_{k}^{-}\right]|k-2, n\rangle \\
& -\lambda \sqrt{(n+1)(n+2)}\left(\mu_{k}^{+}+\nu_{k}^{+}\right)|k, n+2\rangle \\
& +\lambda \sqrt{n(n-1)}\left(\mu_{k}^{-}+\nu_{k}^{-}\right)|k, n-2\rangle, \quad \text { (A1) }
\end{aligned}
$$

where the different parameters are defined as

$$
\begin{aligned}
\mu_{k}^{ \pm} & =\eta\left(\omega_{k}-\omega_{k+1} \mp \Omega\right)^{-1} \mu_{k} \\
\nu_{k}^{ \pm} & =\eta\left(\omega_{k}-\omega_{k-1} \mp \Omega\right)^{-1} \nu_{k} \\
u_{k}^{ \pm} & =\eta\left(\omega_{k}-\omega_{k+2} \mp 2 \Omega\right)^{-1} \mu_{k}^{\prime} \\
v_{k}^{ \pm} & =\eta\left(\omega_{k}-\omega_{k-2} \mp 2 \Omega\right)^{-1} \nu_{k}^{\prime} \\
u_{k}^{0} & =\eta\left(\omega_{k}-\omega_{k+2}\right)^{-1} \mu_{k}^{\prime} \\
v_{k}^{0} & =\eta\left(\omega_{k}-\omega_{k-2}\right)^{-1} \nu_{k}^{\prime} \\
\lambda & =\eta / 2 \Omega,
\end{aligned}
$$

with $\mu_{k}=1-\delta_{k, N}, \nu_{k}=1-\delta_{k, 1}, \mu_{k}^{\prime}=\mu_{k}\left(1-\delta_{k, N-1}\right)$ and $\nu_{k}^{\prime}=\nu_{k}\left(1-\delta_{k, 2}\right)$

\section{APPENDIX B: EXCITONIC COHERENCES}

The approximate expression of the effective exciton propagator up to second order in the exciton-phonon coupling $V$ is written as

$$
\begin{aligned}
G_{k_{2} k_{1}}(t) & =\frac{Z_{B}^{k_{1}}(t)}{Z_{B}} e^{-i \hat{\omega}_{k_{1}} t}\left[1-\left(n_{k_{1}}(t)+1\right)\left(\mu_{k_{1}}^{+2}+\nu_{k_{1}}^{+2}\right)-n_{k_{1}}(t)\left(\mu_{k_{1}}^{-2}+\nu_{k_{1}}^{-2}\right)\right] \delta_{k_{2}, k_{1}} \\
& +\frac{Z_{B}^{k_{1}+1}(t)}{Z_{B}} e^{-i \hat{\omega}_{k_{1}+1} t}\left[e^{i \hat{\Omega}_{k_{1}+1} t} n_{k_{1}+1}(t) \nu_{k_{1}+1}^{+2}+e^{-i \hat{\Omega}_{k_{1}+1} t}\left(n_{k_{1}+1}(t)+1\right) \nu_{k_{1}+1}^{-2}\right] \delta_{k_{2}, k_{1}} \\
& +\frac{Z_{B}^{k_{1}-1}(t)}{Z_{B}} e^{-i \hat{\omega}_{k_{1}-1} t}\left[e^{i \hat{\Omega}_{k_{1}-1} t} n_{k_{1}-1}(t) \mu_{k_{1}-1}^{+2}+e^{-i \hat{\Omega}_{k_{1}-1} t}\left(n_{k_{1}-1}(t)+1\right) \mu_{k_{1}-1}^{-2}\right] \delta_{k_{2}, k_{1}} \\
& +\frac{Z_{B}^{k_{1}}(t)}{Z_{B}} e^{-i \hat{\omega}_{k_{1}} t} u_{k_{1}}^{0}\left[\left(n_{k_{1}}(t)+1\right) \mu_{k_{1}}^{+}+n_{k_{1}}(t) \mu_{k_{1}}^{-}\right] \delta_{k_{2}, k_{1}+2} \\
& +\frac{Z_{B}^{k_{1}}(t)}{Z_{B}} e^{-i \hat{\omega}_{k_{1}} t} v_{k_{1}}^{0}\left[\left(n_{k_{1}}(t)+1\right) \nu_{k_{1}}^{+}+n_{k_{1}}(t) \nu_{k_{1}}^{-}\right] \delta_{k_{2}, k_{1}-2} \\
& +\frac{Z_{B}^{k_{2}}(t)}{Z_{B}} e^{-i \hat{\omega}_{k_{2}} t} v_{k_{2}}^{0}\left[\left(n_{k_{2}}(t)+1\right) \nu_{k_{2}}^{+}+n_{k_{2}}(t) \nu_{k_{2}}^{-}\right] \delta_{k_{2}, k_{1}+2} \\
& +\frac{Z_{B}^{k_{2}}(t)}{Z_{B}} e^{-i \hat{\omega}_{k_{2}} t} u_{k_{2}}^{0}\left[\left(n_{k_{2}}(t)+1\right) \mu_{k_{2}}^{+}+n_{k_{2}}(t) \mu_{k_{2}}^{-}\right] \delta_{k_{2}, k_{1}-2} \\
& +\frac{Z_{B}^{k_{1}+1}(t)}{Z_{B}} e^{-i \hat{\omega}_{k_{1}+1} t}\left[e^{i \hat{\Omega}_{k_{1}+1} t} n_{k_{1}+1}(t) \mu_{k_{1}+1}^{+} \nu_{k_{1}+1}^{+}+e^{-i \hat{\Omega}_{k_{1}+1} t}\left(n_{k_{1}+1}(t)+1\right) \mu_{k_{1}+1}^{-} \nu_{k_{1}+1}^{-}\right] \delta_{k_{2}, k_{1}+2} \\
& +\frac{Z_{B}^{k_{1}-1}(t)}{Z_{B}} e^{-i \hat{\omega}_{k_{1}-1} t}\left[e^{i \hat{\Omega}_{k_{1}-1} t} n_{k_{1}-1}(t) \mu_{k_{1}-1}^{+} \nu_{k_{1}-1}^{+}+e^{-i \hat{\Omega}_{k_{1}-1} t}\left(n_{k_{1}-1}(t)+1\right) \mu_{k_{1}-1}^{-} \nu_{k_{1}-1}^{-}\right] \delta_{k_{2}, k_{1}-2}
\end{aligned}
$$

where $n_{k}(t)=\left[\exp \left(\beta \Omega+i \delta \Omega_{k}^{(2)} t\right)-1\right]^{-1}$. At time $t=$ $0, n_{k}(t=0)$ reduces to the average phonon number at thermal equilibrium $\bar{n}=[\exp (\beta \Omega)-1]^{-1}$.

\section{APPENDIX C: FOURTH ORDER ENERGY CORRECTION}

To third order, the energy correction $\epsilon_{k, n}^{(3)}$ vanishes due to the symmetry of the coupling Hamiltonian $V$. By 
contrast, the fourth order energy correction is written as

and

$$
\epsilon_{k, n}^{(4)}=\delta \omega_{k}^{(4)}+n \delta \Omega_{k n}^{(4)}
$$

where $\delta \omega_{k}^{(4)}$ and $\delta \Omega_{k n}^{(4)}$ are the fourth order correction to the exciton energy and to the phonon energy, respectively. They are defined as

$$
\begin{aligned}
\delta \omega_{k}^{(4)} & =2 \eta\left(\mu_{k}^{+2} u_{k}^{+}+\nu_{k}^{+2} v_{k}^{+}\right)-2 \eta \lambda\left(\mu_{k}^{+}+\nu_{k}^{+}\right)^{2} \\
& +\eta\left(\mu_{k}^{+2} u_{k}^{0}+\nu_{k}^{+2} v_{k}^{0}\right) \\
& -\eta\left(\mu_{k}^{+2}+\nu_{k}^{+2}\right)\left(\mu_{k}^{+}+\nu_{k}^{+}\right)
\end{aligned}
$$

$$
\begin{aligned}
\delta \Omega_{k, n}^{(4)} & =\eta(n+3)\left(\mu_{k}^{+2} u_{k}^{+}+\nu_{k}^{+2} v_{k}^{+}\right)+\eta(n-1)\left(\mu_{k}^{-2} u_{k}^{-}+\nu_{k}^{-2} v_{k}^{-}\right)-\eta \lambda(n+3)\left(\mu_{k}^{+}+\nu_{k}^{+}\right)^{2}+\eta \lambda(n-1)\left(\mu_{k}^{-}+\nu_{k}^{-}\right)^{2} \\
& +\eta u_{k}^{0}\left[(n+2) \mu_{k}^{+2}+n \mu_{k}^{-2}+2(n+1) \mu_{k}^{+} \mu_{k}^{-}\right]+\eta v_{k}^{0}\left[(n+2) \nu_{k}^{+2}+n \nu_{k}^{-2}+2(n+1) \nu_{k}^{+} \nu_{k}^{-}\right] \\
& -\eta(n+2)\left(\mu_{k}^{+2}+\nu_{k}^{+2}\right)\left(\mu_{k}^{+}+\nu_{k}^{+}\right)-\eta n\left(\mu_{k}^{-2}+\nu_{k}^{-2}\right)\left(\mu_{k}^{-}+\nu_{k}^{-}\right)-\eta(n+1)\left(\mu_{k}^{-2}+\nu_{k}^{-2}\right)\left(\mu_{k}^{+}+\nu_{k}^{+}\right) \\
& -\eta(n+1)\left(\mu_{k}^{+2}+\nu_{k}^{+2}\right)\left(\mu_{k}^{-}+\nu_{k}^{-}\right)
\end{aligned}
$$

Eq.(C3) shows that the energy correction of a single phonon is not well-defined due to the nonlinear dependence of $n \delta \Omega_{k, n}^{(4)}$ versus the phonon number $n$. To overcome this difficulty, a mean field procedure is applied. To proceed, the effective energy of a single phonon $\hat{\Omega}_{k}$ is defined according to the relation $\left\langle n \Omega+n \delta \Omega_{k}^{(2)}+n \delta \Omega_{k, n}^{(4)}\right\rangle=$ $\bar{n} \hat{\Omega}_{k}$, where the symbol $\langle\ldots\rangle$ is an average over the phonon number according to the density matrix $\rho_{B}$. The correction of the phonon energy up to fourth order is finally expressed as $\delta \Omega_{k}^{(4)}=\left\langle n \delta \Omega_{k, n}^{(4)}\right\rangle / \bar{n}$. It is easily extracted from Eq.(C3) by using the relation $\left\langle n^{2}\right\rangle=2 \bar{n}^{2}+\bar{n}$.
* Electronic address: vincent.pouthier@univ-fcomte.fr

1 A. S. Davydov, Theory of Molecular Excitons (McGrawHill Book Co., New York, 1962).

2 V. May and O. Kuhn, Charge and Energy Transfer Dynamics in Molecular Systems (Wiley-VCH Verlag, Berlin, 2000).

3 V. Sundstrom, Prog. in Quantum Electron. 24, 187 (2000).

4 T. Renger, V. May, and O. Kuhn, Phys. Rep. 343, 137 (2001).

5 Th. Renger and R.A. Marcus, J. Chem. Phys. 116, 9997 (2002).

6 M. Schroder, U. Kleinekathofer, and M. Schreiber, J. Chem. Phys. 124, 084903 (2006).

7 M. Mohseni, P. Rebentrost, S. Lloyd, and A. AspuruGuzik, J. Chem. Phys. 129, 174106 (2008).

8 A. S. Davydov and N. I. Kisluka, Phys. Status Solidi 59, 465 (1973); Zh. Eksp. Teor. Fiz 71, 1090 (1976) [Sov. Phys. JETP 44, 571 (1976)].

9 A.C. Scott, Phys. Rep. 217, 1 (1992).

10 V. Pouthier, Phys. Rev. E 68, 021909 (2003).

11 J. Edler, R. Pfister, V. Pouthier, C. Falvo, and P. Hamm, Phys. Rev. Lett. 93, 106405 (2004).

12 C. Falvo and V. Pouthier, J. Chem. Phys. 123, 184709 (2005); 123, 184710 (2005).

13 D. Tsivlin and V. May, Chem. Phys. 338, 150 (2007).

14 P.A.S. Silva and L. Cruzeiro, Phys. Rev. E 74, 021920
(2006)

15 V. Pouthier and Y.O. Tsybin, J. Chem. Phys. 129, 095106 (2008).

16 V. Pouthier, Phys. Rev. E 78, 061909 (2008).

17 V. Pouthier, J. Phys. : Condens. Matter 21, 185404 (2009).

18 V. Pouthier, J. Chem. Phys. 132, 035106 (2010).

19 E. R. Bittner, A. Goj, and I. Burghardt, Chem. Phys. 370, 137 (2010).

20 B.N.J. Persson, Phys. Rev. B 46, 12701 (1992)

21 V. Pouthier and C. Girardet, Phys. Rev. B 60, 13800 (1999).

22 M. Bonn M, C. Hess, and M. Wolf, J. Chem. Phys. 115, 7725 (2001).

23 V. Pouthier, J.C. Light, and C. Girardet, J. Chem. Phys. 114, 4955 (2001).

24 P. Jakob, J. Chem. Phys. 114, 3692 (2001).

25 V. Pouthier and C. Girardet, Phys. Rev. B 65, 035414 (2002).

26 V. Pouthier, J. Chem. Phys. 118, 3736 (2003).

27 W.G. Roeterdink, O. Berg, and M. Bonn, J. Chem . Phys. 121, 10174 (2004).

28 V. Pouthier, Phys. Rev. B 71, 115401 (2005).

29 V. Pouthier, Phys. Rev. B 74, 125418 (2006).

30 C. M. Tesch and R. de Vivie-Riedle, Phys. Rev. Lett. 89, 157901 (2002).

31 C. Tesch and R. de Vivie-Riedle, J. Chem. Phys. 121, 
$12158(2004)$

32 C. Gollub and R. de Vivie-Riedle, Phys. Rev. A 78, 033424 (2008).

33 C. Gollub, Ph.D. thesis, Ludwig Maximilian University of Munich, 2009

${ }^{34}$ H. Fröhlich, Adv. Phys. 3, 325 (1954).

35 T. Holstein, Ann. Phys. (N.Y.) 8, 325 (1959); 8, 343 (1959).

${ }^{36}$ M. Schlosshauer, Decoherence and the Quantum-toClassical Transition (Spinger Verlag, Berlin, 2007).

37 H.P. Breuer and F. Petruccione, The theory of open quantum systems (Oxford University Press, New York, 2007).

38 F. Shibata, Y. Takahashi, and N. Hashitsume, J. Stat. Phys. 17, 171 (1977).

39 C. Uchiyama and F. Shibata, Phys. Rev. E 60, 2636 (1999).

40 H.P. Breuer and B. Kappler, Annals of Physics 291, 36 (2001).

41 H.P. Breuer, J. Gemmer, and M. Michel, Phys. Rev. E 73, 016139 (2006).

42 A. Pereverzev and E. Bittner, J. Chem. Phys. 125, 104906 (2006).

43 S.M. Barnett and S. Stenholm, Phys. Rev. A 64, 033808 (2001).

44 V. Pouthier, J. Phys. : Condens. Matter 22, 255601 (2010).

45 V. Pouthier, Phys. Rev. B 80, 144304 (2009).

46 V. Pouthier, Phys. Rev. E 75, 061910 (2007)

47 V. Pouthier, Phys. Rev. E 81, 031913 (2010).

48 V. Pouthier, J. Phys. : Condens. Matter 22, 385401 (2010).

49 H.P. Breuer, Phys. Rev. A 75, 022103 (2007).

50 J. Fischer and H.P. Breuer, Phys. Rev. A 76, 052119 (2007).

51 E. Ferraro, H.P. Breuer, A. Napoli, M.A. Jivulescu, and A. Messina, Phys. Rev. B 78, 064309 (2008).

52 C.R. Willis and R.H. Picard, Phys. Rev. A 9, 1343 (1974).

53 T. Koide, Prog. Theo. Phys. 107, 525 (2002).

54 O. Linden and V. May, Physica A 254, 411 (1998).

55 K.H. Hughes, C.D. Christ, and I. Burghardt, J. Chem. Phys. 131, 024109 (2009).
56 K.H. Hughes, C.D. Christ, and I. Burghardt, J. Chem. Phys. 131, 124108 (2009).

57 D. Wang, L. Chen, R. Zheng, L. Wang, and Q. Shi, J. Chem. Phys. 132, 081101 (2010).

58 G. Nan, Q. Shi, and Z. Shuai, J. Chem. Phys. 130, 134106 (2009).

59 Y. Tanimura and R. Kubo, J. Phys. Soc. Jpn. 58, 101 (1989).

60 Y. Tanimura, Phys. Rev. A 41, 6676 (1990).

61 A. Ishizaki and Y. Tanimura, J. Phys. Soc. Jpn. 74, 3131 (2005).

62 J. Piilo, S. Maniscalco, K. Harkonen, and K.-A. Suominen, Phys. Rev. Lett. 100, 180402 (2008).

63 P. Rebentrost, R. Chakraborty, and A. Aspuru-Guzik, J. Chem. Phys. 131, 184102 (2009).

64 J. Piilo, S. Maniscalco, K. Harkonen, and K.-A. Suominen, Optics and Spectroscopy 108, 407 (2010).

65 L. D. Landau and E. M. Lifshitz, Quantum Mechanics (Pergamon Press, Ltd., London, 1958).

66 M. Wagner, Unitary Transformations in Solid State Physics (North-Holland, Ansterdam, 1986).

67 S. Mukamel, Principles of Nonlinear Optical Spectroscopy (Oxford University Press, New York, 1995).

68 S. Bose, Phys. Rev. Lett. 91207901 (2003).

69 J. Wang and J. Feng, Protein Engineering 16, 799 (2003).

70 R. Bloem, A. G. Dijkstra, T. la Cour Jansen, and J. Knoester, J. Chem. Phys. 129, 055101 (2008).

${ }^{71}$ K. Moritsugu, O. Miyashita, and K. Kidera, Phys. Rev. Lett. 85, 3970 (2000)

72 H. Fujisaki, Y. Zhang, and J.E. Straub, J. Chem. Phys. 124, 144910 (2006).

73 N. Ostergard, in Davydov Soliton Revisited, edited by P. L. Christiansen and A. C. Scott (Plenum, New York, 1990), pp 229.

74 R. Bluhm and V. A. Kostelecky, and J.A. Porter, Am. J. Phys. 64, 944 (1996). 